\title{
Plastic instability studied experimentally on a semi-crystalline polymer through thermomechanical heat source identification:
}

\author{
the flow stress concept revisited.
}

\author{
Jing YE, Stéphane ANDRE ${ }^{\dagger}$, Laurent FARGE, Nicolas LOUVET \\ Laboratoire d'Energétique et de Mécanique Théorique et Appliquée (LEMTA), \\ UMR 7563 CNRS, University of Lorraine \\ 2 avenue de la Forêt de Haye, TSA 60604-54518 Vandoeuvre-Lès-Nancy, France \\ ${ }^{\dagger}$ Corresponding author: \\ e-mail: stephane.andre@univ-lorraine.fr
}




\begin{abstract}
Micromechanical deformation phenomena such as those leading to macroscopic viscoelastic and plastic behavior must be studied from a thermodynamic viewpoint, as they induce complex and partly irreversible heat effects. Calorimetric measurements of the intrinsic volumetric Thermomechanical Heat Sources (THS) activated in the material bulk during mechanical loads can produce valuable information with respect to that aim. They can be based on infrared imaging if submitted to inverse algorithms that allow a correct reconstruction of THS to be produced. Here, an inverse method relying on a diffusion-advection heat transfer model is applied to experimental temperature maps recorded during tensile tests. These are made on a semi-crystalline polymer that shows a strong development of plastic instabilities. Along with simultaneous kinematic observables produced with a Digital Image Correlation system, the competition between advection and diffusion phenomena may be clearly established. 1-D profiles of the reconstructed THS and measured strain rates illustrate clearly that thermomechanical effects associated with necking onset and propagation follow the kinematic variable in a rather direct manner. Finally, we show for tensile experiments that THS estimations lead to analyze plasticity as a rheological behavior controlled by the flow stress, responsible of necking development and propagation.
\end{abstract}

Keywords: Thermomechanical Heat Source, Inverse methods, Plastic instability, Semi-Crystalline Polymers, Flow stress. 


\section{Introduction}

Plastic instabilities correspond to sudden phenomena associated to a strong transition of rheological regime. To quote the definition given by Coussot (2018), one could consider that they refer to "(i) a transition from a solid regime to a flowing regime which does not correspond to a true "liquid state," the flow in this regime may rather be seen as a succession of solid states during very large deformation; (ii) a strong tendency to localization of the yielded regions in some small region of the material while the rest of the material undergoes some deformation in its solid state". This description is in full agreement with experimental facts observed on semi-crystalline polymers over decades. But the physical phenomena related to the plastic instabilities are still to be fully understood and described, in particular to make any commercial Finite Element software that solves the mechanical stress/strain problem more predictive.

Scientific studies of the rheological behavior of solid materials involving plastic instabilities should normally favor a thermodynamic approach as they are always accompanied with dissipation. This is required especially because strong thermomechanical couplings occur, which result from a direct coupling between the dependent variables of the considered thermodynamic potential considered. With this aim in mind, many experimental studies have been conducted for years on a considerable number of different materials to measure calorimetric effects activated only by mechanical stress and strain variables (Tauchert and Afzal, 1967; Adams and Farris, 1988; Chrysochoos and Louche, 2000; Bhalla et al., 2003). In the last 3 decades, this field of research has received much attention thanks to the widespread availability of Infra-Red (IR) imaging cameras. A lot of studies go no further than the analyzis of local temperature evolutions, to gain insight into the physical mechanisms at stake (Maj et al., 2012). These studies remain qualitative, as temperature is not intrinsic to what happens in the bulk Representative Elementary Volume (REV) and thus proscribes any rheophysical understanding. The only way to advance in the direction of a real thermodynamically based study is to proceed to a reconstruction of the so-called Thermomechanical Heat Sources (THS). In general, this requires a strong commitment to the field of mathematical inverse methods, as is seen for example in Aufray et al. (2000), although shortcut analyses are sometimes used (Chrysochoos and Louche, 2000; Doudard et al., 2010; Toussaint et al., 2012; Delpueyo et al., 2013). Many inverse methods have been put forward to reconstruct unknown source terms from 
the heat diffusion operator, either in the bulk of the material, at its boundaries, or corresponding to unknown initial conditions. Fewer methods are available when advection is considered along with diffusion (Rap et al., 2007; Maalej et al., 2012; Souza and Roberty, 2012). This was necessary here as the development of plastic instabilities in polymers is responsible for strong kinematic effects. Everything presented in this paper is thus the result of a long process decomposed into three steps. A first paper (Ye et al., 2015) presents detailed results of the kinematic phenomenon through precise measurements made with 3-D stereo Digital Image Correlation (DIC). The plastic instability initiation, development and propagation are analyzed there through the local velocity or strain rate observables, expressed in either a Lagrangian or Eulerian framework. A second paper (Ye et al., 2016), is rather theoretical and gives all detailed information and bibliographic background concerning the mathematical method used to perform inverse reconstruction of the THS from IR recordings of temperature maps and velocity measurements. These were in fact synthetic data, produced (i) with an idealized model of the thermal transfer in a specimen for a well-known idealized mathematical form of THS and (ii) with idealized velocity profiles, but with the same characteristic profiles as those measured during real experiments (Ye et al., 2015). Hence, the current report, which is the last paper in this series of three, aims to show the results that the inverse algorithm produces on real velocity and temperature data. Monitoring of the THS in both space and time variables can then be analyzed to identify different thermomechanical regimes in relation to the development of plastic instability. The paper is organized as follows:

In the next section, the experimental set-up used to produce the data is briefly described. In section "Thermodynamic Heat Transfer Modeling", we return to the thermodynamic foundations used in this study to derive the state laws, i.e. the behavior law of our material, which has both a rheological and a thermal component. The latter, through an entropy balance, naturally leads to the heat equation used for THS inversion. Our knowledge of the order of magnitudes of local velocities in plastic instabilities and of the associated dimensionless Peclet number suggests considering a reconstruction that accounts for the influence of both diffusion and advection. The Inverse Problem Formulation is then presented. In the "Thermomechanical observations from inversion" section, experimental results of reconstructed THS are presented. These are discussed according to various possible options of the inversion process (linked to various possible model assumptions). Elements are provided that allow the reader to measure 
the difficulties inherent to this goal and the performances reached in this study. Lastly, the rheological implications that can be drawn from the THS measurements are discussed. The main finding of our work is that a flow stress can be calculated from the local THS measurements, as a function of the local strains and local strain rates. This "thermo-mechanically determined" flow stress is shown to correspond fairly well to the mechanical flow stress that can be reached from tensile-relaxation tests. This supports the simple idea that the THS associated with the plastic flow is essentially the sole contribution of heat dissipation.

\section{Experimental setup}

Experimental platform This is fully described in Andre et al. (2007) and Ye et al. (2015). It consists of a servo-hydraulic MTS ${ }^{\circledR} 810$ load frame surrounded by three metrological components (Fig.1):

- A homemade Video-extensometry system allows the axial or longitudinal true strain $\varepsilon_{y}^{V E}(y \approx 0)=\ln \left(l_{y} / l_{0_{y}}\right)$ to be measured in situ and in real-time in the middle of our specimen $(y \approx 0)$. Thanks to this external signal, the tensile machine is controlled to perform tensile tests at constant imposed strain rates $\dot{\varepsilon}_{y}^{V E}(y \approx 0)=\dot{\varepsilon}_{0}^{V E}$. As a consequence, most of the results presented below are indexed on the true strain $\varepsilon_{0}^{V E}$.

- A stereo 3-D DIC system (Aramis3D), which provides full field measurements of the displacement vector on the specimen and consequently full field strain tensor maps $\underline{\underline{\varepsilon}}(x, y, t)$ or longitudinal profiles. These are measured on the opposite face of the specimen with respect to the Video-extensometer real-time monitoring of the central strain. As will be seen later, the system provides the local velocity measurements necessary for this study.

- An IR camera (JadeIII-SC7000 from Cedip/Flir systems), which provides the temperature fields $T(x, y, t)$.

The DIC and IR equipments work alternatively rather than simultaneously, for two different tests on two different specimens. This can introduce a bias when comparing thermal and kinematic measurements and therefore makes it crucial to have the exact same ramp command 
$\dot{\varepsilon}_{y}^{V E}(y \approx 0)=\dot{\varepsilon}_{0}^{V E}$ realized by the set-up when tests are repeated to produce sets of DIC and IR movies.

Fig. 1 also shows the geometry of the specimen, which is designed in such a way that the onset of plasticity takes place precisely at its center (REV of uniform cross-section).
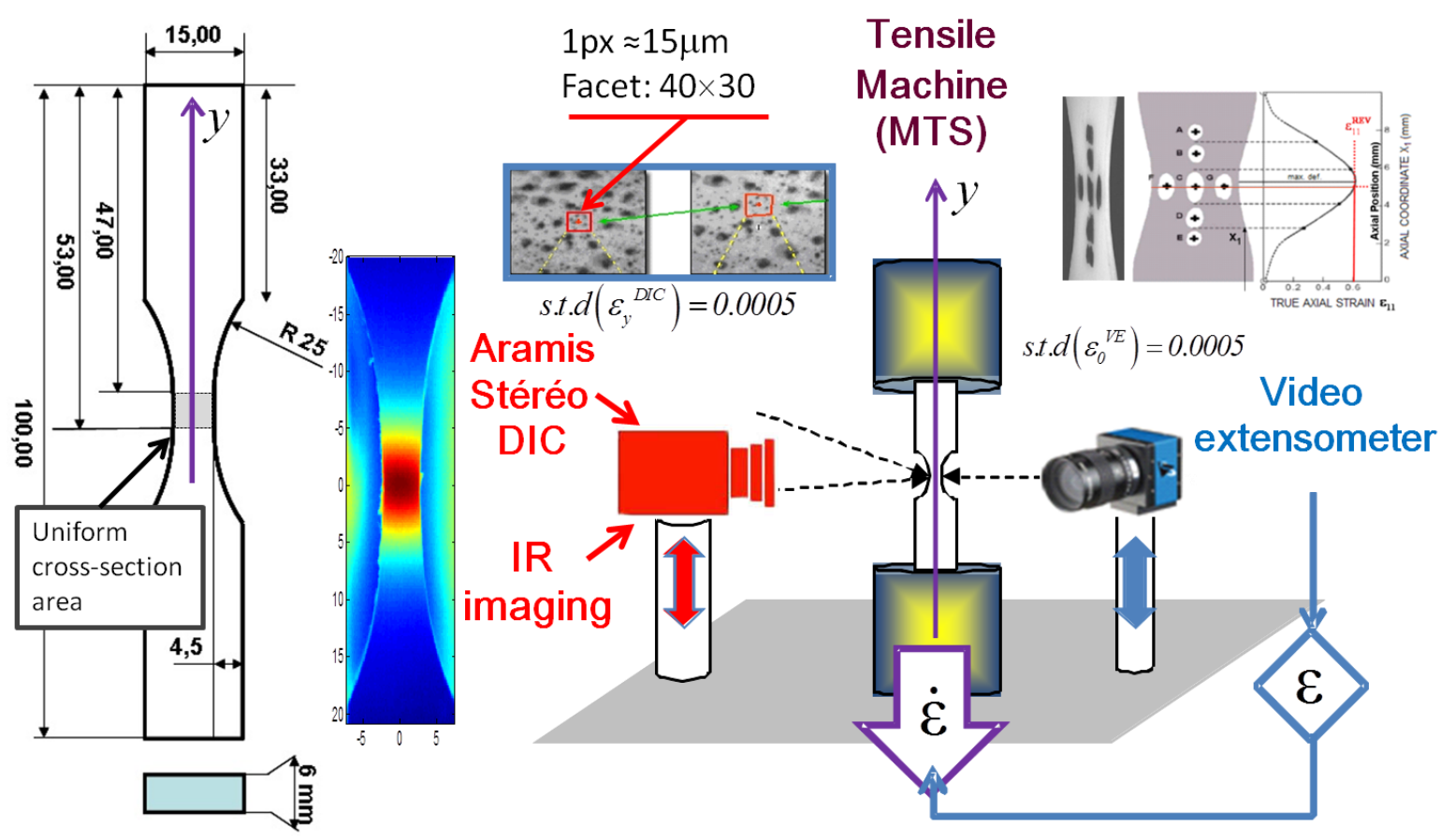

Fig. 1 Sketch of the experiment and geometry of the specimen (left)

Material investigated Specimens are made of High Density PolyEthylene (HDPE) produced by Röchling Engineering Plastics KG ( "500 Natural”" grade), a material studied for more than 15 years in our laboratory. It has been characterized at all scales in the $\AA$ to $\mu \mathrm{m}$ range, with multiple physical investigations of the microstructure and also at the macroscopic scale (Farge et al., 2015; Ye et al., 2015; Blaise et al., 2016).

Experimental conditions Previous experiments of the same type aiming to measure THS were performed at low strain rates, with an inverse model based solely on the diffusion phenomenon (Andre et al., 2012). The metrological objective in that case is a hard challenge to achieve: at low strains, heat effects are of small magnitude (quasi non measurable for strain rates below 
$\left.0.005 \mathrm{~s}^{-1}\right)$. The signal-to-noise ratio of recorded temperatures is not very high and applying an inverse method to such data produces THS outputs of poor quality. In this paper we aimed to investigate higher strain rates. This provides thermomechanical effects of greater amplitudes, hence better THS outputs. However, there are drawbacks; the high local strain rates occurring with the onset of plasticity require both the development of an inverse model taking into account heat advection and the provision of velocity measurements as additional inputs of the inverse process. This was the original purpose of this research. Four imposed strain rates are considered for the experiments: $\dot{\varepsilon}_{0}^{V E}=0.01 \mathrm{~s}^{-1}, \dot{\varepsilon}_{0}^{V E}=0.02 \mathrm{~s}^{-1}, \dot{\varepsilon}_{0}^{V E}=0.03 \mathrm{~s}^{-1}, \dot{\varepsilon}_{0}^{V E}=0.04 \mathrm{~s}^{-1}$. To reach a maximum deformation of $\varepsilon_{y}(y \approx 0)=2$ in the most stretched central part of the specimen then requires between 50 and 200 seconds. Reaching higher strain rates is difficult because the controller PID settings of the actuator/feedback loop are not able to produce a perfectly defined strain rate ramp behavior. Higher strain rates also change the nature of the problem; different physical/rheological behavior of the material and simpler identification models such as an adiabatic assumption become relevant.

\section{Thermodynamic Heat Transfer Modeling}

Thermodynamic framework In the context of Thermodynamics of Irreversible Processes (T.I.P.), the rheological behavior of a REV of some material is obtained in a thermodynamically consistent way by writing state laws. These clearly express all the couplings between macroscopic and internal dual variables, the latter being in charge of describing the multiscale evolution of the microstructure and making the intrinsic heat dissipation calculation possible. According to Callen (1985) they are obtained by stating that the internal energy is a potential which contains all information about the system, even in out-of-equilibrium situations. Highly detailed information is available from previous research (Andre et al., 2012; Cunat et al., 2001) and we reiterate only the basic outline and principles, which are sufficient to allow the reader to grasp the subject.

The volume-specific internal energy is given from Euler fundamental equation, summing its thermal ( $T, s$ variables), mechanical ( $\sigma, \varepsilon$ variables) and chemical $\left(\mu_{k}, n_{k}\right.$ variables) contributions 


$$
u\left(s, \underline{\underline{\varepsilon}}, z^{j}\right)=T s+\underline{\underline{\sigma}}: \underline{\underline{\varepsilon}}+\sum_{j} A^{j} z^{j}
$$

where a first but important step has already been made, which consists of substituting the classical $\left(\mu_{k}, n_{k}\right)$ dual chemical variables for the affinity-extent of the reaction $\left(A^{j}, z^{j}\right)$ dual variables (see Prigogine, 1949; and Callen, 1985 for affinities as generalized forces; Nowick and Berry, 1972; and Meixner, 1954 for affinities as conjugates of normal internal variables). This is a classical procedure (Kuiken, 1994), but one can insist on the fact that, for the mechanical scientist, it allows realization of the scale transition. Various $j$-type processes are considered which involve the $k$-species in unknown chemical reactions. $A^{j}$ is the affinity associated with process $\mathrm{j}$ representing a non-equilibrium generalized force, which allows the perturbed microstructure to proceed to an equilibrium recovery. Within this point of view, the state laws are straightforward (Tisza, 1966; Cunat, 2001) and lead to generalized matrix form, such as for example the following one when the Helmholtz potential is considered:

$$
\left[\begin{array}{c}
-\dot{s} \\
\dot{\sigma} \\
-\dot{\dot{A}}
\end{array}\right]=\left(\begin{array}{ccc}
-\frac{\rho C}{T} & -\alpha E & \bar{b}_{t h} \\
-\alpha E & E & \bar{b}_{\text {mech }} \\
{ }^{t} \bar{b}_{t h} & { }^{t} \bar{b}_{\text {mech }} & \bar{g}
\end{array}\right)\left[\begin{array}{c}
\dot{T} \\
\dot{\varepsilon} \\
\overline{\dot{z}}
\end{array}\right]
$$

In view of the 1-D tensile test, the mechanical tensor quantities $\underline{\underline{\sigma}}, \underline{\underline{\varepsilon}}, \underline{\underline{\underline{E}}}$ are replaced by scalar ones and $E$ corresponds to the rigidity or Young modulus.

The upper bar stands for tensorial quantities linked to internal variables (the collection of $j-$ type processes gathered in the $\bar{z}$ vector). $\bar{b}_{t h}, \bar{b}_{\text {mech }}$ stand for the coupling matrix between the macroscopic observable variables and the internal, non-observable variables. For this matrix form of state laws to be operational, the $\underline{\bar{b}} \cdot \bar{z}$ products, which represent the non-elastic or dissipative component of the entropy and stress responses of eqs. $2 \mathrm{a}, 2 \mathrm{~b}$, are further elucidated, but this aspect will not be detailed here. The interested reader can refer for example to (Blaise et al., 2016; Andre et al., 2012) to learn more about this part of the theory. $\bar{g}$ stands for the coupling matrix between internal variables. In classical reversible thermodynamics, matrices $\underline{b}$ and $\bar{g}$ vanish and one recovers from (2) the state laws associated with the pure thermo-elastic material. 
In view of reconstructing the THS responsible for the temperature field of the specimen, the entropy balance has to be written which, in accordance with T.I.P. treatises (Kuiken, 1994; Munster, 1966), gives equation (3):

$$
\dot{s}=\dot{s}_{\text {exchanged }}+\dot{s}_{\text {internal production }}=-\frac{1}{T} \nabla \cdot \mathbf{J}_{q}+\dot{s}_{i}^{\text {dissipation }}
$$

with

- $\quad \dot{s}_{i}^{\text {dissipation }}=\frac{1}{T} \bar{A} \overline{\dot{z}}:$ dissipation is the flux-force product of internal variables that reflect the non-equilibrium state;

- $\quad \mathbf{J}_{q}$ is the heat flux density composed in our case of both diffusive (Fourier's law) and advection components that is $\mathbf{J}_{q}=-k \nabla T+(\rho c T) \mathbf{V}$ (k: thermal conductivity, $\rho c T$ : volume-specific enthalpy, $\mathbf{V}$ : local velocity).

Taking the negative of equation (2a) as left-hand term of eq.(3) and rearranging terms gives the local heat equation

$$
\rho c \dot{T}=-\nabla \cdot \mathbf{J}_{q} \underbrace{-\alpha E T \underline{\underline{\dot{\varepsilon}}}+T \underline{\bar{b}}_{t h} \overline{\dot{z}}+\bar{A} \overline{\dot{z}}}_{q_{T H S}}=-\nabla \cdot \mathbf{J}_{q}+q_{T H S}
$$

which states that the local enthalpy rate varies according to heat exchanges with the surroundings $\mathbf{J}_{q}\left(W \cdot m^{-2}\right)$ and the THS source term $q_{T H S}\left(W \cdot m^{-3}\right)$. This latter will be identified from the reconstruction process and can be further decomposed according to its three components:

- Reversible thermo-elastic effect $-\alpha E T \underline{\dot{\varepsilon}}$, remaining as long as the tensile test runs;

- Irreversible intrinsic heat dissipation $\mathcal{D}=\bar{A} \overline{\dot{z}} \geq 0$

- Recoverable entropic couplings $T \bar{b}_{t h} \overline{\dot{z}}$ requiring a model that describes the behavior of the internal processes.

\section{Dimensional analysis and considerations concerning the inverse estimation of THS}

Rewriting eq. (4) in a differential and usual form and reducing it to the 1-D case gives 


$$
\underbrace{\rho c \frac{\partial T}{\partial t}}_{\text {accumulation }}+\underbrace{\rho c V \frac{\partial T}{\partial y}}_{\text {advection }}=k \frac{\partial^{2} T}{\partial y^{2}}+q_{\text {THS }}-q_{\text {heat_loss }}
$$

where the $q_{\text {heat_loss }}$ term figures the heat losses in the two transverse directions, as a result of the reduction in the dimension of the problem and non-adiabatic nature of the system. In practice, the time constant for such heat losses is very long compared to the experimental duration and the specimen is confined in an enclosure with reflecting walls. These will therefore be neglected in the treatment, keeping in mind a possible overestimation of the identified $q_{\text {THS }}$.

The second point to discuss concerning equation (5) lies in the necessity or not of accounting for both diffusion and advection for the THS reconstruction, especially when plastic instabilities occur. A preliminary answer is made possible by a precise measurement of local velocities during this phenomenon. These have been obtained using the DIC system and for tensile tests performed at four different imposed strain rates, as described in the Experimental conditions section. As an example (see Fig. 2), typical experimental velocity profiles show large differences with the deformation state (characterized through the $\varepsilon_{0}^{V E}$ strain) and the localization effect in the $[-1 ; 1] \mathrm{mm}$ centered area (Lagrangian reference) starting at $\varepsilon_{y}=0.12$ when yielding starts. All these measurements are available (see Ye et al., 2015). They gave the numerical data used in the dimensional analysis presented below to estimate orders of magnitude of the different mechanisms of heat transfer to be compared. 


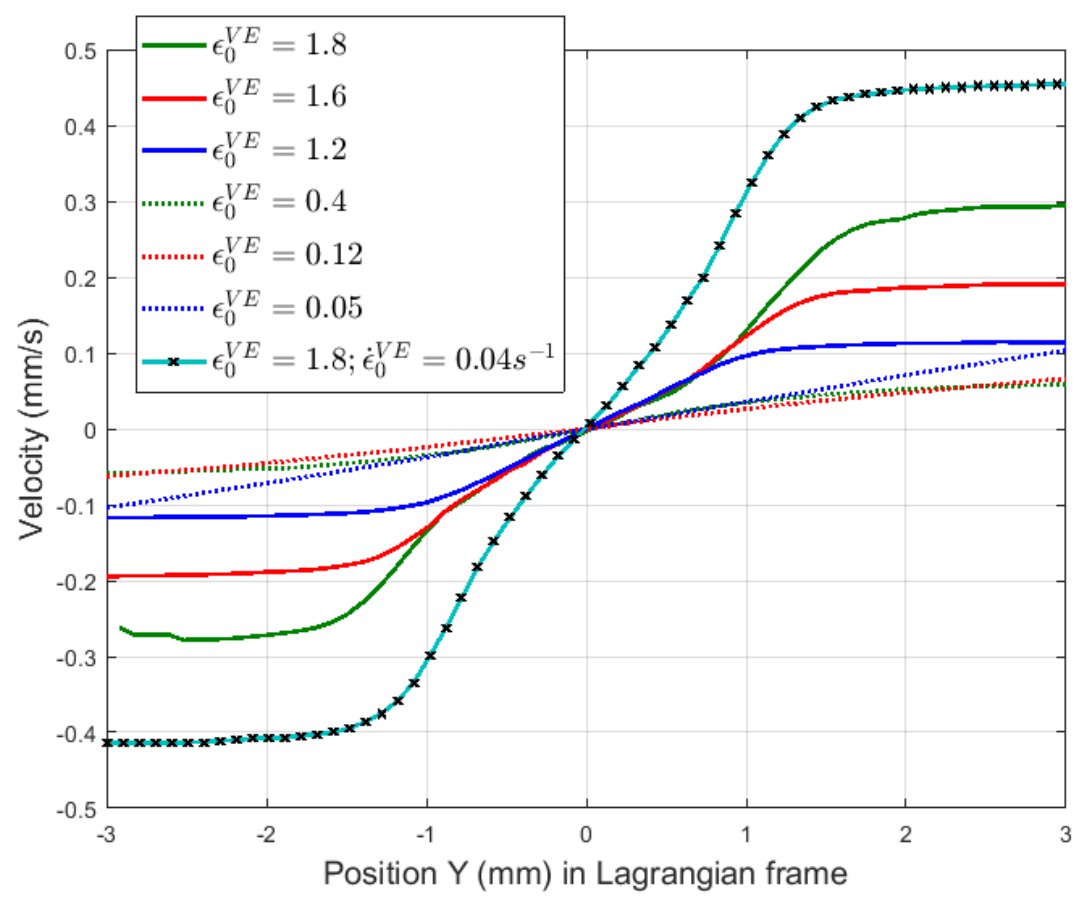

Fig. 2 Velocity profiles versus material (lagrangian) coordinates for different strains $\varepsilon_{0}^{V E}$ obtained for a test at a strain rate of $\dot{\varepsilon}_{0}^{V E}=0.02 \mathrm{~s}^{-1}$. Additional curve for $\varepsilon_{0}^{V E}=1.8$ and $\dot{\varepsilon}_{0}^{V E}=0.04 \mathrm{~s}^{-1}$.

The advection enthalpy flux corresponds to $(\rho c \Delta T) V$ with $\rho c$ the volume heat capacity, $V$ the local velocity, and $\Delta T$ a temperature difference. The diffusive flux is estimated from Fourier's law, $k \Delta T / \ell$ where $k$ is the thermal conductivity, $\ell$ the characteristic length over which diffusion is considered, and $\Delta T$ the same considered difference. The material properties $\rho c$ and $k$ (or thermal diffusivity $a$ ) have been assumed to be constant over the bulk material to obtain eq.(5). Advection can be neglected with respect to diffusion when the ratio of these two remains small, i.e. the Peclet number $P e=\frac{c V \Delta T}{k \Delta T / \ell}=\frac{V \ell}{a}<<1$. The Peclet number is also the 
ratio of the characteristic time of diffusion to the time of propagation $P e=\frac{\ell^{2} / a}{\ell / V}=\frac{t_{a}}{t_{V}}<<1$. The order of magnitude of the three different variables $V, \ell, a$ has to be estimated:

- The thermal diffusivity coefficient is a material parameter. It has been precisely measured by a flash experiment developed in our laboratory: $a=0.275 \mathrm{~mm}^{2} \cdot \mathrm{s}^{-1}$.

- The characteristic diffusion length $\ell$ is estimated as the minimum (metrological) length allowing for a good thermal gradient resolution from IR images. It has been found empirically in various controlled thermal situations that 4 pixels always allow a good computation of thermal gradients. The resolution of $1 p x \approx 130 \mu m$ in our optical configuration makes the estimation of $\ell^{\min } \approx 550 \mu \mathrm{m}$.

- Regarding velocities, it must be pointed out that the local velocity at the center of the specimen is always equal to 0 by symmetry. The maximum absolute velocity is always obtained symmetrically at the extremities of the specimen. The decrease in velocity magnitude can be seen as soon as necking proceeds $\left(\varepsilon_{0}^{V E}=0.12\right)$. As a consequence, the Peclet number will vary along the tensile test.

From this information, Peclet numbers can be estimated for the different regimes, along with typical characteristic times, and the following analysis brings a physical understanding of what can be expected:

- At small strains $(\varepsilon<0.05)$, and according to the investigated strain rates, the experimental time at which to consider the influence of both mechanisms of transfer remains below $5 \mathrm{~s}$. During this time, the typical maximum length affected by diffusion is $\ell=\sqrt{a t}<1 \mathrm{~mm}$. For this length, and the maximum velocity considered, the characteristic time for advection is of the order of $2 \mathrm{~s}$, which means a situation of Peclet approaching unity. At the same time, the THS is only made from the instantaneous 
thermo-elastic effect, which simultaneously affects all parts of the specimen and the dissipative phenomena have not really been triggered. The temperature gradients are then very small, reflecting the nearly homogeneous stress distribution ruled by the sole geometry of the specimen. In consequence, even if both advection and diffusion phenomena have nearly the same impact on heat transfer, this latter remains very weak because the temperature is nearly homogeneous in the specimen. On a REV scale, anywhere in the specimen and considering this short time range, the system appears adiabatic. In other words, the instantaneous thermo-elastic THS is directly responsible for an enthalpy change of the REV.

- When necking initiates ( $\varepsilon_{0} \approx 0.12$ in the center of the specimen), the thermal effect and accompanying temperature gradients appear in the neck, but the velocity field has dropped to very low values due to localization. The investigated local strain rates of $\dot{\varepsilon}=\left[0.01 s^{-1}-0.04 s^{-1}\right]$ and a characteristic length of $\ell \approx 500 \mu m$ lead to velocities of the order of $V=\ell \dot{\varepsilon} \approx[0.005 \mathrm{~mm} / \mathrm{s}-0.02 \mathrm{~mm} / \mathrm{s}]$. These values are perfectly consistent with the velocities of the neck frontiers for this strain level, (see Fig. A-1 in Supplementary material). The diffusion time $t_{a}$ is then of the order of $1 s$ and the local Peclet number ranges from $P e \simeq[0.01-0.04]$, which means that advection is much less efficient than diffusion.

- At the end of the test i.e. for high strains, the two necking shoulders appear as regions with high velocities/high strain rates and non-negligible temperature gradients (Fig.9 in Andre, 2012). A rough estimate of the Peclet number can be made, for example for the strain state $\varepsilon_{0}=1.8$ in the case of $\dot{\varepsilon}=0.04 \mathrm{~s}^{-1}$. The absolute velocities are of the order of $V \approx 0.5 \mathrm{~mm} / \mathrm{s}$ (see Fig. A-2 in Supplementary material) which makes a Peclet number of the order of $P e=V \ell / a \simeq 1$. Advection competes then locally with diffusion and has to be 
considered in the inversion process to produce good estimates of the THS in the shoulders.

This preliminary analysis leads to two important issues for future thermo-rheological investigations of this type:

- The THS reconstructed over the REV at the center of the specimen will always remain insensitive to advection. Due to symmetry, very low velocity magnitudes operate on flat temperature profiles in this area. If one aims only to produce a thermomechanical signal for the purpose of identifying the state laws of a given material (Eqs2), the best strategy is to identify the THS versus time at the center of the specimen in the simpler diffusion case.

- The THS reconstructed when the plastic instability propagates, requires taking into account the advective part. This is unavoidable when one aims to understand the local distribution of the THS with respect to strain rate distributions, a major objective and result in terms of this study.

\section{Inverse Problem Formulation}

This work, which is essentially of a mathematical order, was the object of a separate paper (Ye et al., 2016). A short summary is given here so that the reader can gain insight into the kind of strategy used to build the inversion computational code. The solution to the direct problem (eq.5) was brought about by decomposition of both the temperature and heat source on the same spectral finite basis (dimension N) like for example,

$$
q_{T H S}(y, t)=\sum_{i=1}^{N} d_{i}(t) V_{i}(y)
$$

where the $V_{i}$ 's are the selected spectral modes and the $d_{i}$ 's, the THS coefficients to be estimated as a function of time to reconstruct the heat source. Two algorithms have been 
considered which depends on the choice of a spectral basis: Algorithm A1 uses a very sophisticated spectral basis known as the Branch modes (see Videcoq et al. 2008). It allows considering any kind of boundary conditions. It was specifically used to reconstruct the whole experimental THS profiles on a [-L,L] segment along the y-axis of the specimen. Algorithm A2 uses the more classical Fourier modes. Even if the two algorithms produce close results (Ye et al. 2016), this latter was proved to give slightly more precise results, taking advantage of the obvious physical symmetry of necking which leads to consider only half-profiles of temperature data, with the nice consequence of producing an exact fixed null flux - a null temperature gradient- boundary condition. The cosine family offers then a perfect tool to respect this boundary condition. Additionally, the temperature profile can be averaged over its two mirror-sides and this favors a better signal-to-noise ratio, a determinant parameter in difficult inverse problems. The inversion runs then over temperature profile data along the $[0, \mathrm{~L}]$ $\mathrm{y}$-axis length of the specimen.

For both algorithms, an optimization process has been designed to minimize a least-square criterion $J_{L S}\left(q_{T H S}\right)$ with terminal cost evaluated at final time $t_{f}$, which is actually the norm of the residuals between measured $T(M, t)$ and calculated $T\left(M, t ; q_{T H S}\right)$ temperatures of the specimen:

$$
J_{L S}(q)=\frac{1}{2}\left(\int_{0}^{t_{f}}\left\|T(M, t)-T\left(M, t ; q_{T H S}\right)\right\|^{2} d t\right)+\frac{1}{2}\left\|T\left(M, t_{f}\right)-T\left(M, t_{f} ; q_{T H S}\left(t_{f}\right)\right)\right\|^{2}
$$

Temperatures $T\left(M, t ; q_{T H S}\right)$ are produced by the direct spectral model. The $q_{T H S}$ notation used for the arguments of the temperature variable shall not disturb the reader: the objective is well and truly to identify the decomposition coefficients $d_{i}(t)$. The number $N$ of decomposition coefficients i.e. the dimension of the selected spectral basis can be chosen classically in such a way that it produces a good regularization of the problem, ensuring stability and uniqueness of the solution. 
Because solving this problem requires an estimation of the descent gradient $\nabla J_{L S}\left(d_{i}\right)$ in the case of a large size problem, the method of Lagrange multipliers or of the adjoint state is used in algorithm A2. It is known commonly as the Conjugate Gradient Method. It produces an iterative algorithm (Ye et al. 2016) which converges to the exact solution but, traditionally in inverse problems, may lack stable convergence. To overcome this technical issue, a special feature has been developed during this study. It consists of applying a Truncated Singular Value Decomposition on the Gram matrix of $\nabla J_{L S}$. Such treatment which can be considered as a filtering process on high frequency singular vectors allows the representation of $\nabla J_{L S}$ with the minimum degree of freedom and then the production of a set of descending directions having the best impact on the optimization process. The performance of the estimation can be assessed through the quality achieved in the residuals and has been extensively studied on theoretical cases (Ye et al., 2016). The algorithms that were developed allow the user to choose between:

- two different spectral bases (A1 and A2) used for the temperature/THS decomposition,

- two different boundary conditions in the y-direction (Neuman or Robin type) leading to two different strategies; identification is performed from the whole longitudinal profile or half of it, taking advantage of the symmetry of the problem and the resulting good consequence of averaging the signal on both sides,

- two different initial conditions (real temperature profiles on the specimen or ideally considered as set to a constant "room temperature"),

- two different inverse "engines" (with or without a priori information and different regularization techniques)

The best compromise between these different options was chosen for the inversion of the THS according to our good stabilized initial experimental conditions, the level of noise on the data, the physical insight obtained from the rough observables i.e. symmetry, the quality of the reconstruction (regularization process adapted to our signal to noise ratio). The inversion 
settings remained unchanged for all data inversion and were selected as those leading to the optimal choice from reference results obtained on synthetic noisy data. Results obtained with algorithm A1 will be presented first up to compare THS identifications on whole temperature profiles in order to highlight the differences obtained when neglecting or not the advection phenomenon. Results obtained with algorithm A2 on half profiles will be given starting from section Magnitude of the THS as a function of the strain rate and in the rest of the paper to discuss more quantitatively the physical implications resulting from THS estimations, on the rheological side. 


\section{Thermomechanical observations from inversion: results and discussion}

Diffusive versus Advecto-Diffusive THS reconstructions The first objective of this study was to compare the influence of the two modes of heat transfer in real experimental conditions and for real data. Here we used the full experimental temperature profile with algorithm A1. Fig. 3 shows reconstructed THS profiles in the Eulerian frame for the 4 different imposed strain rates $\dot{\varepsilon}_{0}^{V E}$. The two curves in each plot correspond to the THS reconstruction performed with or without taking into account the advection and associated measured velocity profiles. The spectral approach adopted in the inversion process makes reconstructed THS profiles un-noisy as a result of the spectral approach: once the THS coefficients were identified from the temperature data, eq. (6) computed the linear combination with perfect bases functions. The reconstructions are given for the case of a true strain of 1.8 , which corresponds to a high specimen deformation (around 600\%) for which the neck shoulders had already propagated and the reconstruction biases were the greatest. They made it clear that neglecting or not advection made major differences, with an overestimation of the THS when merely diffusion was considered. These differences were marked at the specimen extremities, whereas it was clear that focusing on the area of pixels at the specimen center made it unnecessary to use a diffusion and advection model for inversion; reconstructions matched perfectly in both cases. This result legitimized a posteriori the thermomechanical results published in Andre (Andre et al., 2012) where the THS was only reconstructed for the central REV and with a pure diffusion model. The existence of an extremity-oriented velocity flattened the temperature profiles: this additional mechanism of transfer favors temperature field homogeneity.

Drawbacks associated with the use of algorithm A1 are clear from the figures, showing THS profiles which lost the symmetry of the problem at high strain rates. Although more or less pronounced, this phenomenon was reproducible on all the experiments we carried out. It 
resulted from high sensitivity of the inversion process to all experimental imperfections, in the first rank of which is the lack of simultaneity of velocity and temperature profile acquisition.
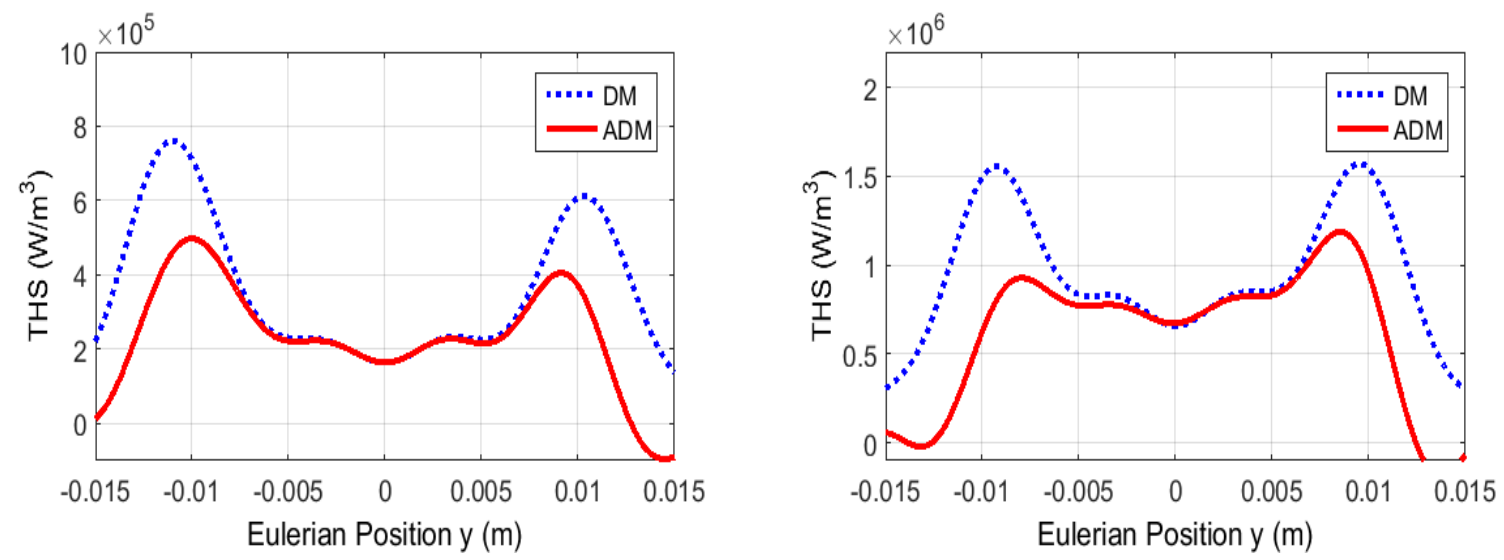

(a) $\dot{\varepsilon}_{0}^{V E}=0.01 \mathrm{~s}^{-1}$

(b) $\dot{\varepsilon}_{0}^{V E}=0.02 \mathrm{~s}^{-1}$
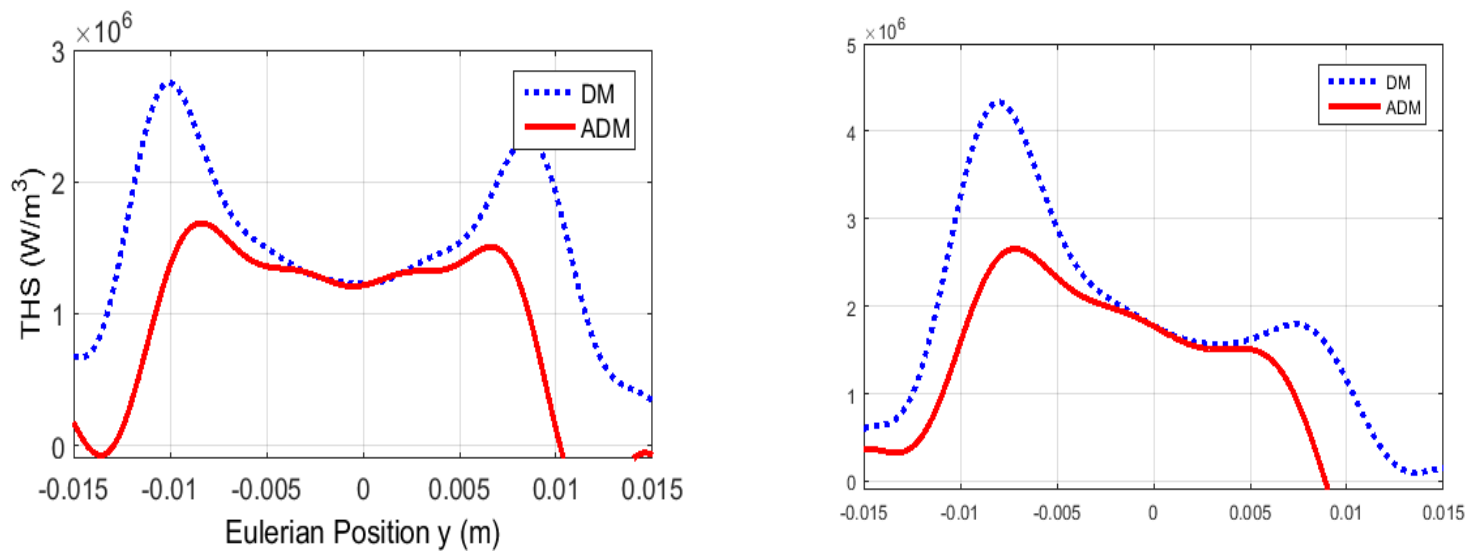

(c) $\dot{\varepsilon}_{0}^{V E}=0.03 s^{-1}$

(d) $\dot{\varepsilon}_{0}^{V E}=0.04 \mathrm{~s}^{-1}$

Fig. 3 Reconstructed THS profiles for the Diffusion Model (DM - Dotted lines) and Advection Diffusion Model (ADM - Solid lines) at a true strain $\varepsilon_{0}^{V E}=1.8$ for different imposed tensile strain rates $\dot{\varepsilon}_{0}^{V E}$.

The same information is reported for a strain of $\varepsilon_{0}^{V E}=0.4$ in Fig.4. Necking was well-established in the central part of the specimen where the gauge length corresponded to 
$-3<y<3 \mathrm{~mm}$. All the strain was localized in this region and a null longitudinal strain was observed outside (see Fig.5c in Ye et al., 2015). The reconstructed THS for all 4 strain rates clearly showed that the THS activity was localized in this region and increased with the strain rate. $\mathrm{ADM}$ or $\mathrm{DM}$ models gave roughly the same reconstruction as expected from the discussion of section 3.2. Slight discrepancies appeared logically where the velocity is the greatest $(y \approx \pm 3 \mathrm{~mm}$ ). Outside of the $[-5 ; 5] \mathrm{mm}$ window, no THS was associated with the null strain state. The temperature there is uniform i.e. there is no temperature gradient and identified THS were very weak. The curve was always wavy at its extremities, with large oscillations as a result of the influence of the first modes on the reconstructions.

Below, we only consider the THS reconstructed using the full advection-diffusive model $(\mathrm{ADM})$.

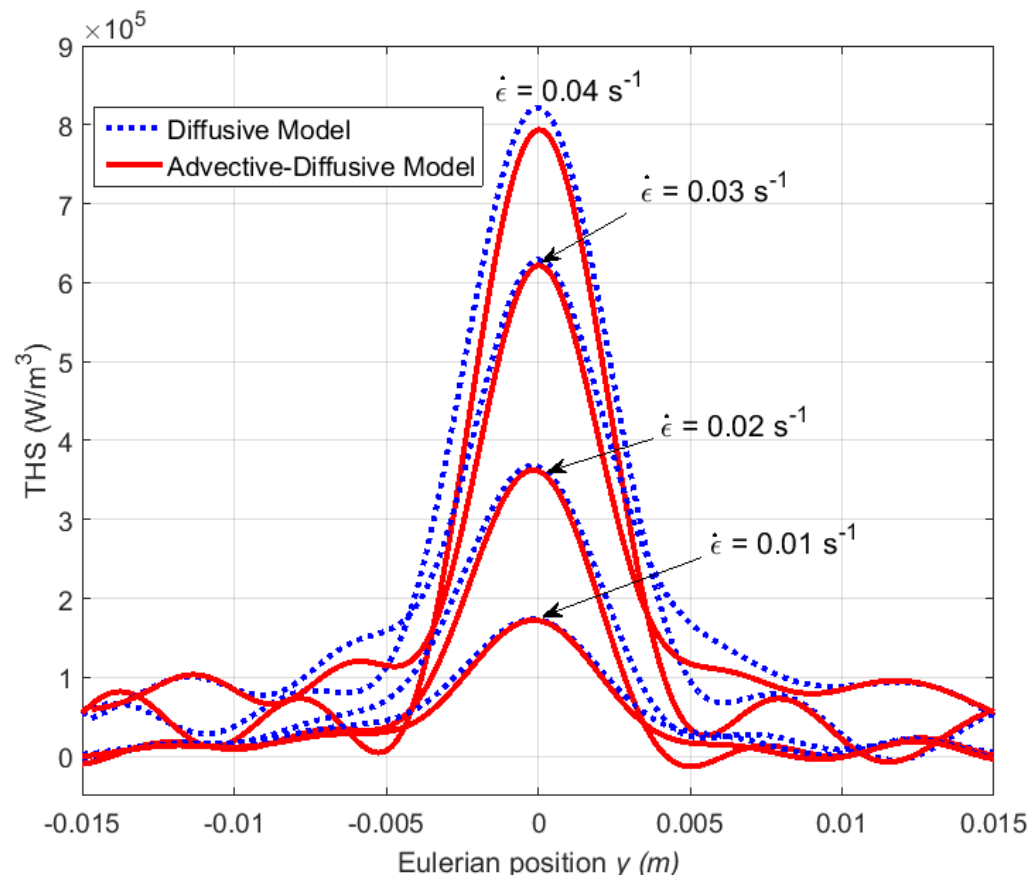

Fig. 4 Reconstructed THS profiles for the Diffusion Model (DM- Dotted lines) and Advection Diffusion Model (ADM- Solid lines) at a true strain $\varepsilon_{0}^{V E}=0.4$ for different imposed tensile strain rates $\dot{\varepsilon}_{0}^{V E}$. 
Thermomechanical observation Fig. 5 presents an example of the temperature field recorded by the IR system along the specimen longitudinal axis and as the tensile test runs. The THS inverse reconstruction proceeds from such a measurement and velocity profile measurements as those reported in Fig. 2 for a few of them. Temperatures during the test range from $22^{\circ} \mathrm{C}$ up to $39^{\circ} \mathrm{C}$ for this imposed strain rate of $\dot{\varepsilon}_{0}^{V E}=0.02 \mathrm{~s}^{-1}$. Not easy to render graphically with the scaling effect is the decrease and non uniformity of the temperature profiles in the early instants (most dark zone) following the initial stage with a uniform temperature profile around $23^{\circ} \mathrm{C}$. This is of course due to the thermoelastic effect which will induce negative THS. This short time non uniformity is simply the result of the non homogenous cross-section along the specimen length. Very rapidly then, the thermomechanical effects produce a raise in temperature which can be seen to be localized first in the central region and further spreads out on the full specimen.

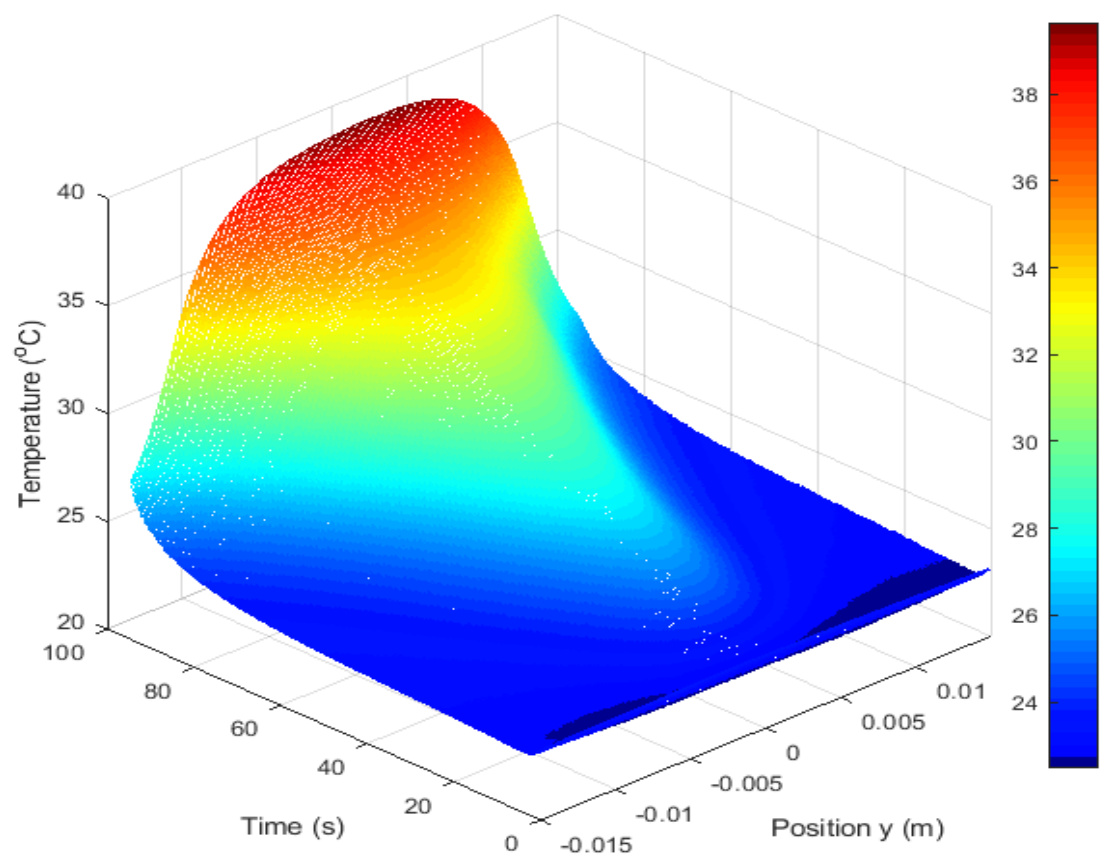

Fig. 5 Temperature map $T(y, t)$ in the Eulerian frame for $\dot{\varepsilon}_{0}^{V E}=0.02 \mathrm{~s}^{-1}$. 
Physical analysis Figures $6 \mathrm{a}$ and $6 \mathrm{~b}$ show the THS reconstructed with algorithm A1 as a function of time and the longitudinal Eulerian $y$ position (Fig.6a) or Lagrangian Y coordinate (Fig.6b). The colorbar shown in Fig.6b applies to Fig.6a as well. A picture of the stretched specimen at the end of the tensile test (time $t=180 \mathrm{~s}$ ) is drawn to clearly show the location of the THS peaks in terms of the laboratory (Eulerian) referential. In fig.6b corresponding to the Lagrangian reference frame (initial configuration), a picture is given of the initially undeformed specimen to emphasize that the THS peaks at the end of the test correspond to material points initially located inside the REV of uniform cross-section. Of course because the specimen is subjected to a very large deformation, the length scales of Figs $6 \mathrm{a}$ and $6 \mathrm{~b}$ cannot be the same. This facilitates the understanding of the plastic instability growth and propagation. At the imposed strain rate of $\dot{\varepsilon}_{0}^{V E}=0.01 \mathrm{~s}^{-1}$, the time axis corresponds directly to the strain $\varepsilon_{y}=\dot{\varepsilon}_{y} t$ in the range [0;2]. The general observed behavior is the same at all imposed strain rates of $\dot{\varepsilon}_{0}^{V E}$ :

- At short times, the THS is negative, with a wide extension over the specimen (Figs.6a and b). It only corresponds to the thermoelastic effect, which is instantaneous and completely reversible. No heat is dissipated ( $\mathcal{D}$ term of $q_{T H S}$ ) nor involved in entropy processes coupled to internal variables. Of course it is non uniform as it obeys the strain-rate distribution which is also non uniform due to the non constant cross-section along the tensile-specimen axis (even before localization takes place).

- The THS activity very quickly becomes positive, simultaneously with the yield stress crossing, which implies a plastic activity. It is then concentrated in a very small area around the specimen center (the necked part, which is approximately shown between the dotted lines of Fig.6b corresponds to a material domain of $4 \mathrm{~mm}$ length, i.e. included in the initial material domain of uniform cross section). The THS magnitude is no longer due solely to the contribution of thermoelasticity, which is still ongoing at the same magnitude, but to additional 
processes provoked by the microstructure transformation such as phase transformation, destruction of the lamellar crystalline structure, dissipation in the amorphous phase, network entropy etc. The THS and strain are both fully localized in this region, as illustrated by a quasi-null THS and strains outside of it.

- As the tensile test progress, the THS activity identifies necking very clearly, firstly as concentrated in the necked gauge part, and secondly when it splits into two, at a characteristic true strain known as the Natural Draw Ratio and of about 1.2 for HDPE. It corresponds to the point where the propagation or extension of the plastic instability requires a new necking development in the shoulders of the specimen, near the grip area remaining undeformed. A section of Fig.6a for the time $t=180 \mathrm{~s}$ ( $\operatorname{strain} \varepsilon_{y}^{V E}=1.8$ ) shows the profiles given in Fig.3a, demonstrating a ratio of THS activity of nearly 2 between the average peak levels and the curve's well level in the central position.

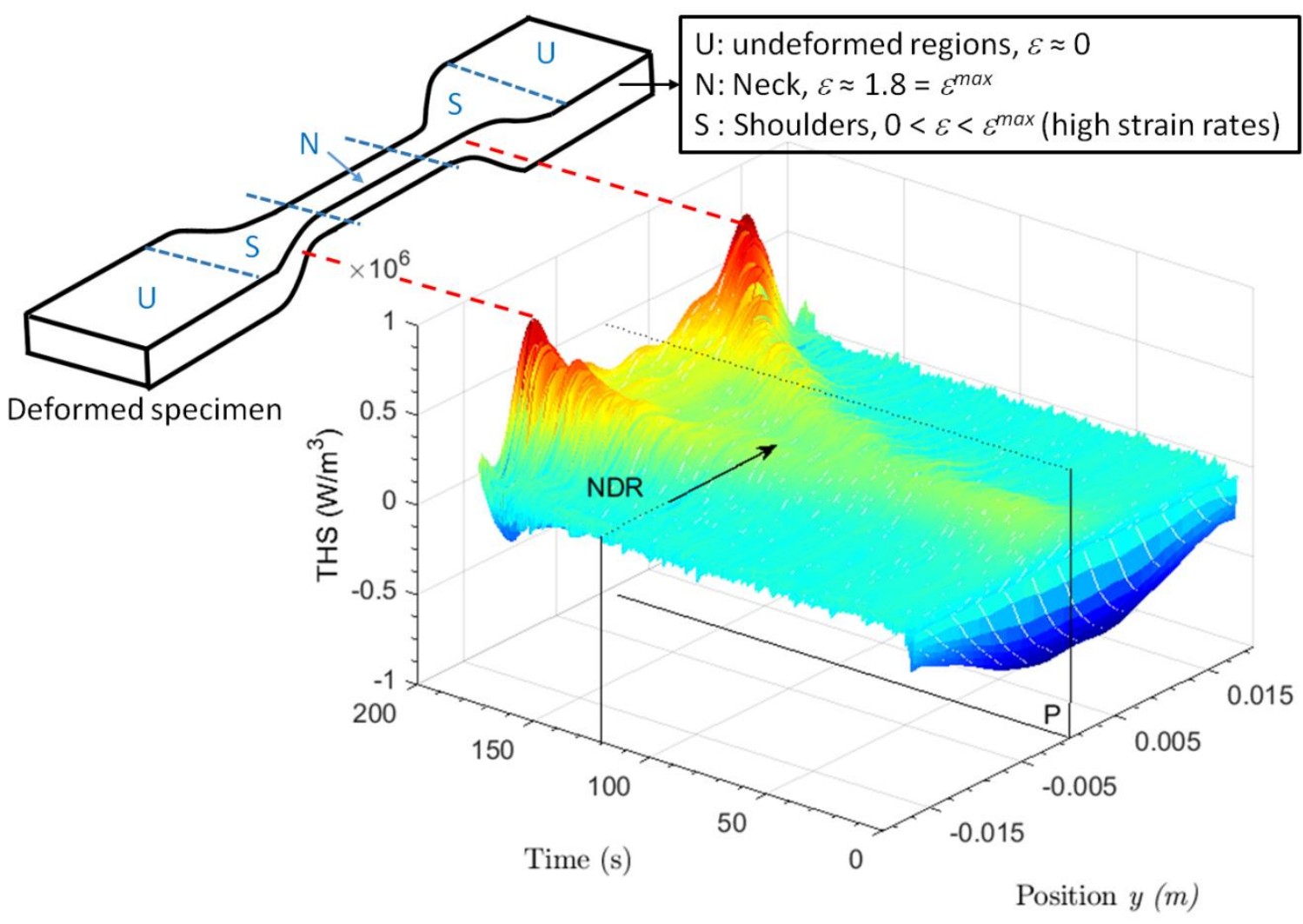

Fig. 6a THS map $q_{T H S}(y, t)$ in the Eulerian frame for $\dot{\varepsilon}_{0}^{V E}=0.01 \mathrm{~s}^{-1}$. 


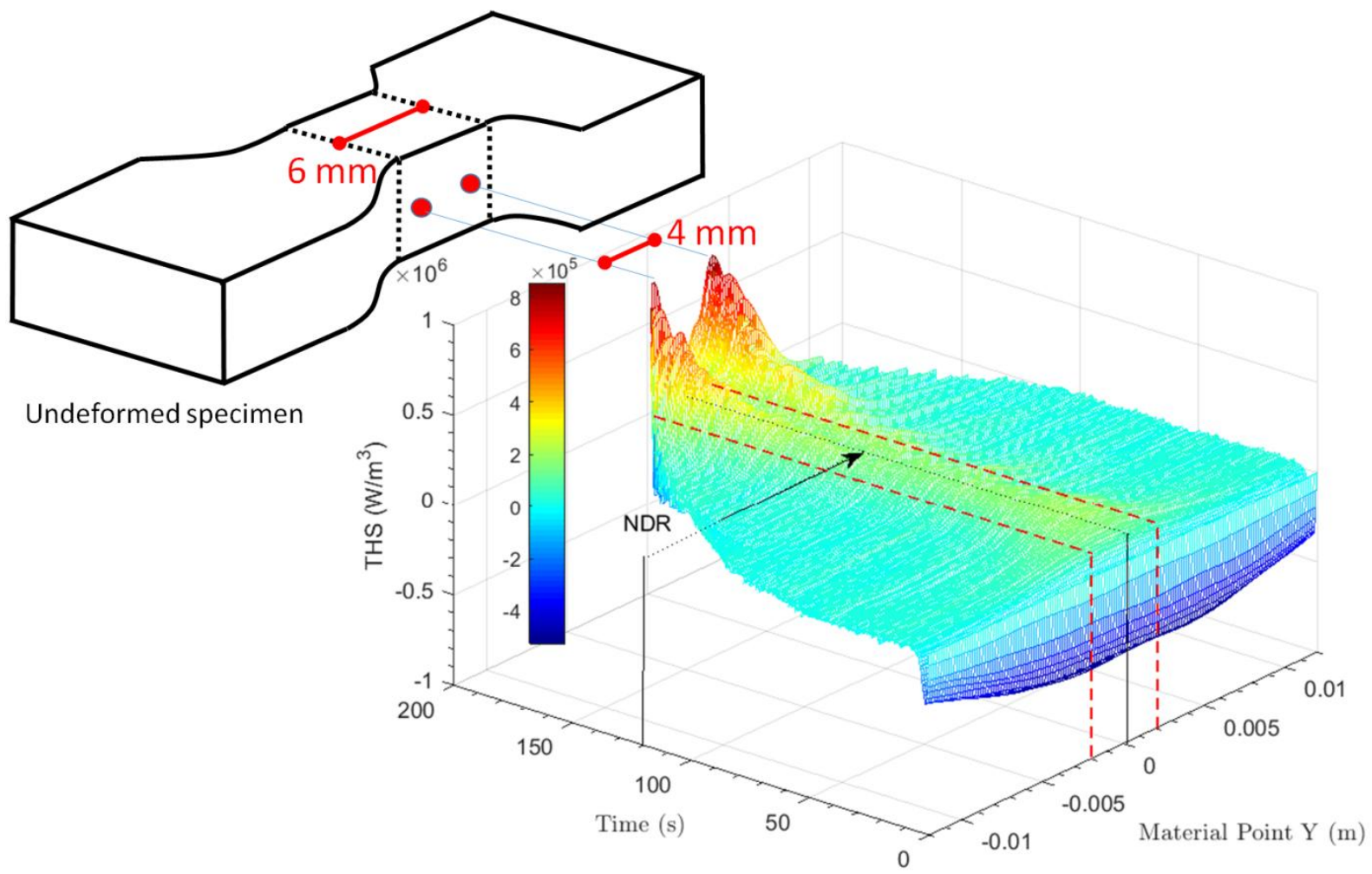

Fig. 6b THS map $q_{T H S}(Y, t)$ in the Lagrangian frame for $\dot{\varepsilon}_{0}^{V E}=0.01 \mathrm{~s}^{-1}$. 
THS and strain rate behaviors Figures $7 \mathrm{a}$ and $\mathrm{b}$ put in parallel strain rate maps $\dot{\varepsilon}_{y}^{D I C}(y, t)$ as measured with the DIC system and the THS $q_{T H S}(y, t)$ as reconstructed from IR thermal images for two different experiments reproduced with the same tensile ramp command $\dot{\varepsilon}_{0}^{V E}=0.01 \mathrm{~s}^{-1}$. In the Lagrangian frame, and as time proceeds, material points having initially been within the camera's field of view exit from it, which explains the lack of information in the left end right upper corners of the Figures. The first observation is that there is a clear agreement between the two observables, which undoubtedly means a simple and direct correspondence between strain rates and THS as necking proceeds. The second consideration is related to clear differences in quality between these two maps, which underline the fact that inversion of temperature data as measured with the best quality cameras available to date appeared unable to compete with data measured in a direct process. However, obtaining DIC measurements in our conditions, where the specimen has a finite width of $2.5 \mathrm{~mm}$ and an extension of nearly $30 \mathrm{~mm}$ is not a straightforward task, as explanations are given in Ye et al. (2015). Despite that, a third conclusion can be drawn, related to the physical behavior of necking in semi-crystalline polymers. The Natural Draw Ratio was defined in the polymer community as the highest draw ratio obtained before the neck began to travel over the whole length of the sample. We showed recently (Ye et al., 2015) that indeed it corresponds to an intrinsic property of the material when measured through full field kinematic information: the strain value at which the central region with the highest magnitude of strain rate splits into two shoulders. The measurement supplies $\varepsilon_{0}^{N D R}=1.2$. It is interesting to note that the Natural Draw Ratio is recovered at the exact same strain on the THS signature, where the THS central region with the maximum activity also splits into two parts (Figs.7a and b). Once again, this indicates more quantitatively that the THS and strain rate fields are fully correlated in the plastic regime. 


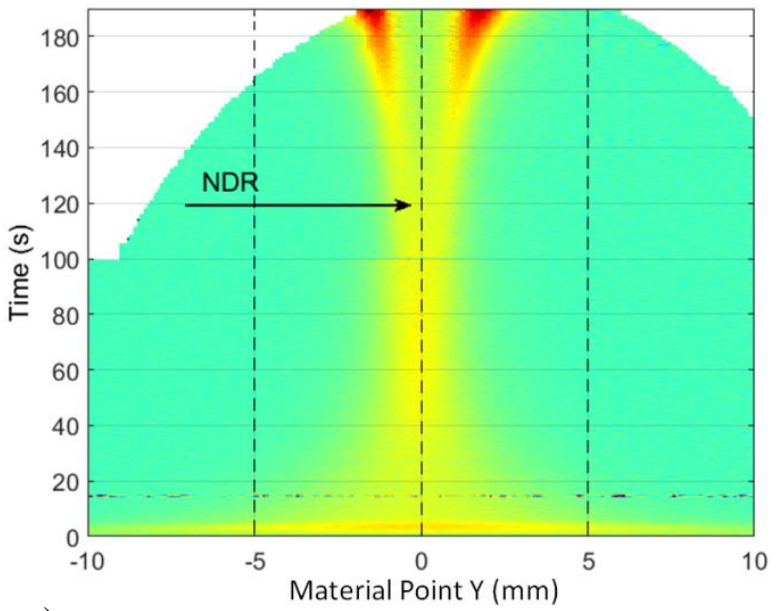

a)

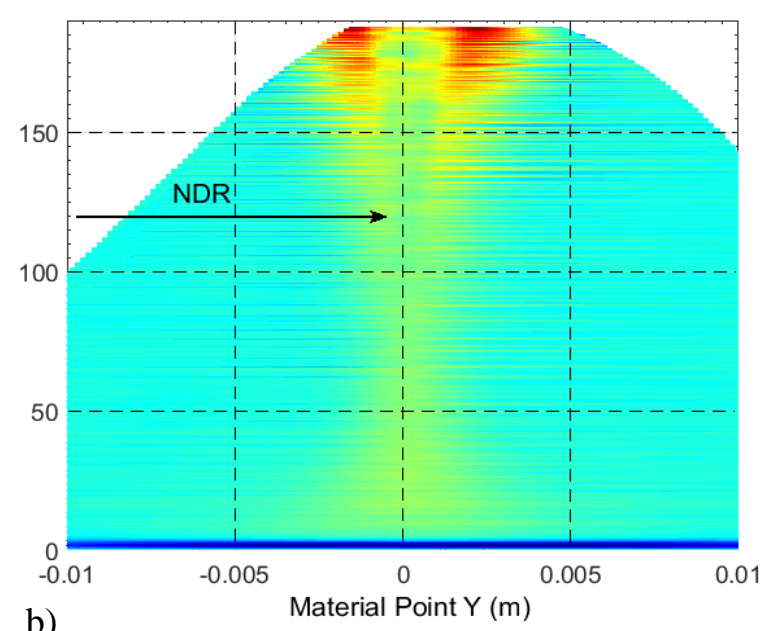

b)

Fig. 7 Strain rate map $\dot{\varepsilon}_{y}^{D I C}(y, t)$ (a) and THS map $q_{T H S}(y, t)$ (b) in the Lagrangian frame for $\dot{\varepsilon}_{0}^{V E}=0.01 \mathrm{~s}^{-1}$

Magnitude of the THS as a function of the strain rate THS measurements have been shown to be difficult to produce, due to a rather complex experimental protocol and an inversion method applied to data of relatively small signal magnitudes. As explained before in the "Inverse Problem Formulation" section, obtaining more precise quantitative conclusions led us to prefer algorithm A2

We first show the THS evolution as a function of time or strain in the middle of the specimen $(y=0)$. This corresponds to the curves obtained from the cutting plane P of Fig.6a. Fig.8 shows the $T H S(\varepsilon)$ curves obtained at the slower strain rate $\dot{\varepsilon}_{0}^{V E}=0.01 s^{-1}$ for 4 repeated experiments. This gives the reader an additional view of the quality of THS estimations obtained in this work. The true stress-true strain curve is shown in the same figure (Right axis) to relate the thermomechanical THS behavior to the purely mechanical one. Thermoelasticity makes the THS decrease instantaneously and this component is kept all along the tensile test (order of magnitude $q_{T H S}^{\text {thermoelastic }}=-\alpha E T \dot{\varepsilon}_{0}^{V E} \approx-5.510^{5} \mathrm{~W} \cdot \mathrm{m}^{-3}$ ) so that the sign inversion of the THS is a clear manifestation of a positive heat source accompanying the plastic instability 
onset. The plateau reached for $\varepsilon_{0}^{V E} \approx 0.2$ on the mechanical curve corresponds to the maximum of the THS curve from which a regime at rather a constant heat source proceeds. It remains roughly constant until it rises from the above-mentioned $\varepsilon_{0}^{N D R}$ (Natural Draw Ratio) where additional heat effects come into play with the hardening regime.

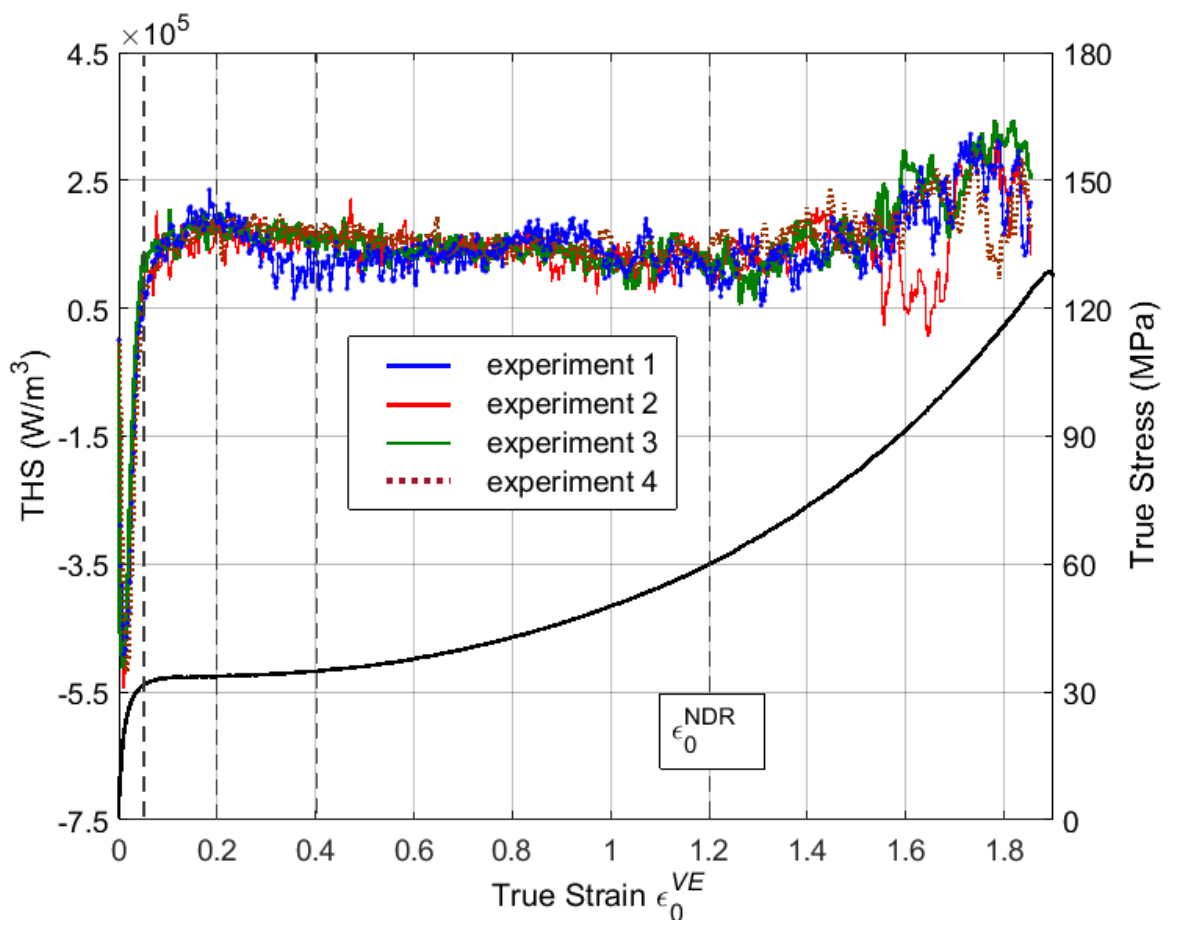

Fig. 8 THS (Left axis) and True Stress (Right axis) versus strain at the specimen's center. Reproducibility is as found in 4 repeated experiments and reconstructions at $\dot{\varepsilon}_{0}^{V E}=0.01 \mathrm{~s}^{-1}$

Fig.9 gives the four $T H S(\varepsilon)$ curves obtained for the four imposed $\dot{\varepsilon}_{0}^{V E}$ strain rates. The same trends can be observed. According to eq.(4), the thermoelastic effect is more intense with proportional strain rates. The hardening is accompanied by more intense heat effects with strain rates. A clear overshoot is now obtained at the yield stress and the onset of plastic instability. The oscillations behind this point again show a small experimental artefact. For strain rates greater than $0.01 \mathrm{~s}^{-1}$, a problem of reactivity of the feedback loop to ensure a constant strain rate $\dot{\varepsilon}_{0}^{V E}$ from the real-time local measurements of the Video-extensometer exists. The best PID 
settings of the feedback loop cannot avoid very small strain oscillations due to the actuator displacement, which result in large amplitudes of the THS signal due to the contribution of the large thermoelastic effects. This problem increases when the strain rate level is raised, but as it corresponds to an instantaneous and reversible effect, it does not affect the average behavior. We do not intend to further investigate here a well-defined phenomenon such as thermoelasticity, but one could imagine correction of the THS curve oscillations from the recorded effective strain rate signal in relation to $q_{T H S}^{\text {thermoelastic }}=-\alpha E T \dot{\varepsilon}_{y}$.

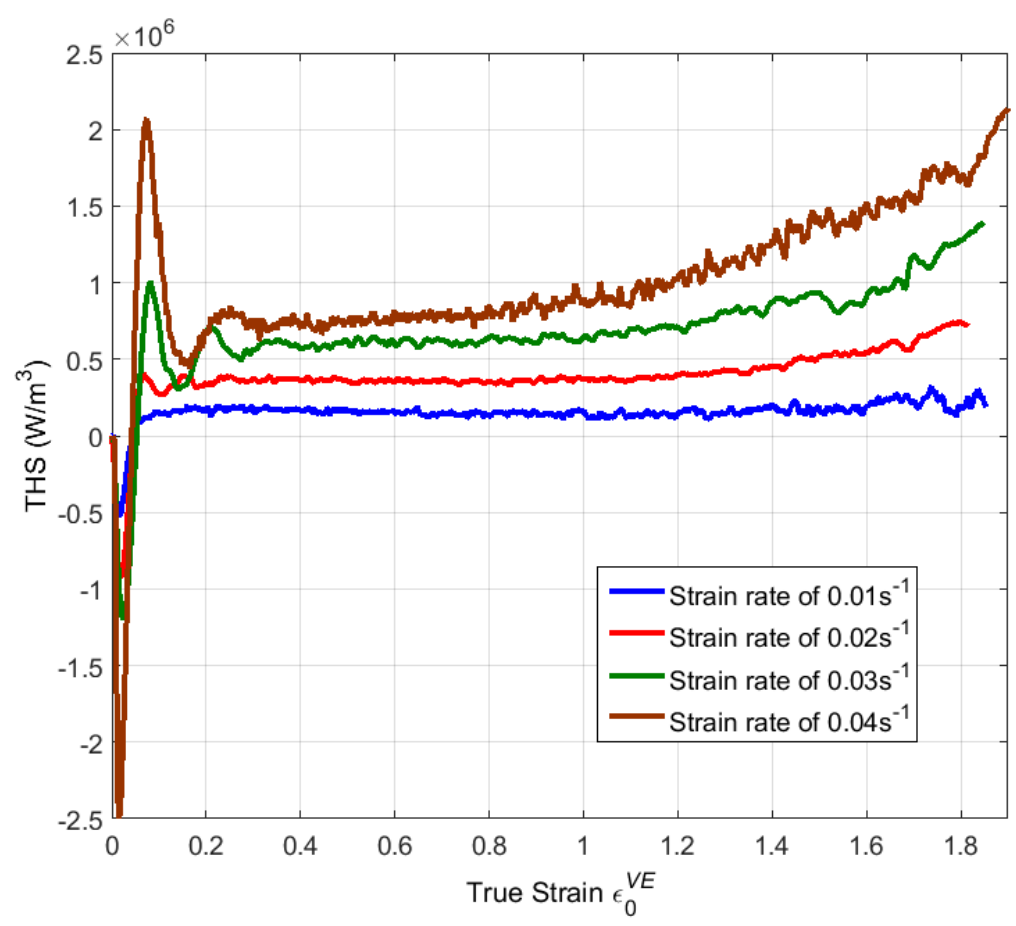

Fig. 9 THS versus strain at the neck and specimen center for different strain rates.

An alternative to investigate trends accompanying the THS magnitude and strain rates relies on the joint analysis of both observable profiles along the specimen axis as shown in Fig.10 for $\dot{\varepsilon}_{0}^{V E}=0.01 s^{-I}$ (Fig.10a) and $\dot{\varepsilon}_{0}^{V E}=0.03 s^{-I}$ (Fig.10b) and for five selected strain states $\left(\varepsilon_{0}^{V E}=0.12 / 0.4 / 1.4 / 1.6 / 1.8\right)$. These profiles (dashed lines for $\dot{\varepsilon}_{y}$ and solid lines for THS) correspond to horizontal (temporal) cuttings from Figs.7 (a and b). As a result of using 
algorithm A2, only half-profiles are shown. These are plotted versus the Lagrangian coordinate and in mirror symmetry with respect to the 0 position (specimen center) to highlight the good correspondence between both kinematic and thermal signals during the onset, development and propagation of necking.

The following general behavior was observed:

- Single-peak regime: strain range was $0<\varepsilon_{0}^{V E}<1.2 \approx \varepsilon_{0}^{N D R}$. The strain rate profile evolved from a wide bell-shaped curve before necking initiation ( $\varepsilon=0.05, \varepsilon=0.12$ ), to a peaked curve at necking onset ( $\varepsilon=0.4$ see also Fig.4). This was further deformed with a flat top $\left(\varepsilon \approx 1.2=\varepsilon_{0}^{N D R}\right)$ before necking propagation. The maximum of these curves for the strain rate profiles (dashed lines) is in the specimen center and more or less respects the imposed strain rate (Left axis horizontal line).

- Double-peak regime: strain range was $\varepsilon_{0}^{V E} \geq 1.2 \approx \varepsilon_{0}^{N D R}$. The rheological hardening regime clearly corresponded to the propagation of the neck in the two symmetrical shoulders. It was marked by the appearance of two peaks in both the THS and strain rate profiles and these double-peak curves were clearly visible at $\varepsilon=1.4,1.6,1.8$, showing an enhanced activity for microstructural transformation in the shoulders, when the central strain rate $\dot{\varepsilon}_{0}^{V E}$ remains imposed. In the centre of the specimen, the THS and strain-rates were approximately constant with a clear extended plateau when profiles were plotted in Eulerian coordinates (See Fig.B in Supplementary material). Such 'M' profiles as a signature of a microstructural activity in the shoulders have been also obtained though the measurement of a void fraction from X-ray microtomography carried out on a PVDF semi-crystalline polymer (Rosenberg et al., 2011). 

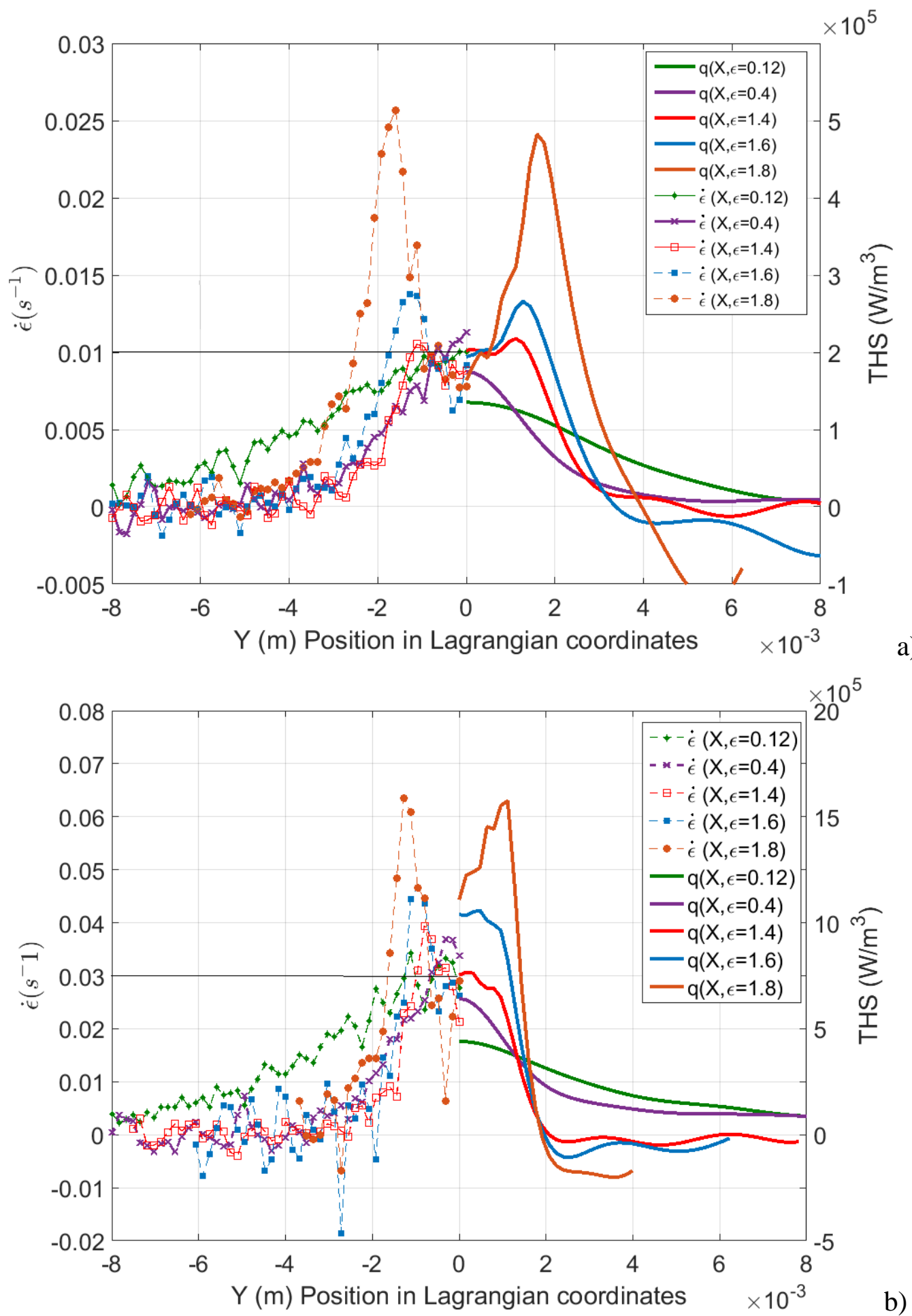

Fig. 10 Strain rate (Left axis) and THS (Right axis) profiles with respect to the Lagrangian position $\mathrm{Y}(\mathrm{m})$ for 5 selected strain rates. a) Tensile test at $\dot{\varepsilon}_{0}^{V E}=0.01 \mathrm{~s}^{-1}$, b) at $\dot{\varepsilon}_{0}^{V E}=0.03 \mathrm{~s}^{-1}$. 


\section{Rheological investigation: a thermomechanical-based flow stress estimation}

From all the local data (THS and strain rate measurements), we attempted to obtain a quantitative insight into the general behavior of the THS versus strain rate and associated rheological outcomes. For this, we relied on the conclusions of a previous study (Andre et al., 2012), which made use of the thermodynamic model of section 3 and showed in all likelihood that the thermoelastic and entropic components of the THS balance one another out in the steady state regime, i.e. when necking is established and plasticity governs deformation. The consequence was that the measured THS roughly corresponded to the irreversible dissipated power: $T H S \cong \mathscr{D}=\bar{A} \overline{\dot{z}}$. The question to attempt to answer is: does the THS or intrinsic power $\mathcal{D}$ behave as proportional to $\dot{\varepsilon}$ as a result of a solid friction type of behavior, as has been widely discussed when considering extensional flows of melt polymer systems? Or alternatively can it be rather related to $\dot{\varepsilon}^{2}$ as a result of a fluid-like behavior and viscous effect? In this section, the local true strain rate is denoted simply by $\dot{\varepsilon}$, and refers to precise measurements obtained by DIC.

Measurements made in the specimen neck center In Fig.11 the local measurements of THS versus $\dot{\varepsilon}$ obtained in the specimen neck center $(y=0)$ are shown, for five different strain states (Curves A,B,C,D,E). The curves correspond to the following cases:

- Curve A : $\varepsilon=\varepsilon^{\max }=0.3$, neck development single-peak regime;

- Curve B : $\varepsilon=\varepsilon^{\max }=1.2$, single-peak regime, just before neck propagation;

- Curve C : $\varepsilon=\varepsilon^{\text {plateau }}=1.4$, initiation of the double-peak regime;

- Curves D and E : $\varepsilon=1.6$ and 1.8 respectively, full double-peak regime. 
The solid line curves correspond to the linear regression of each data set (prime notation). The THS is measured directly from Fig. 9, with appropriate interpolation of the curves, or from profiles such as those shown for example in Fig.10, where averaging of a series of profiles taken before and after a given $\varepsilon$ is necessary because of the noisy character of the reconstructed THS. The associated error bars were evaluated by accounting for the discrepancies observed on repeated experiments. The first comment is that the THS or dissipated power not only depends on $\dot{\varepsilon}$ but also on the strain level. In a recent paper reporting on the full scanning of a specimen undergoing tension during synchrotron Small Angle X-ray Scattering real-time experiments (Farge et al., 2017), it was shown that the local strain univocally governs the microstructural state. It is then normal to observe an intrinsic dissipation which directly depends on the underlying microstructural state. Regarding the strain rate dependency and accounting for a significant measurement uncertainty, the global trend seems to be a linear dependency.

Measurements made in the specimen shoulders To go further, we can take advantage of our local measurements and add additional points to obtain another range of strain rates. This is possible when measuring the maximum THS recorded in the double-peak regime (for the imposed central strain measurement $\varepsilon=1.8$ ). In the neck shoulders where THS is at its maximum, DIC gives a local strain measurement of only $\varepsilon=1.3$, which falls between the two selected strain states of plots B and C. Nevertheless, within that regime, the associated local strain rates jump towards higher values, giving additional interesting points. For the four tensile tests performed, at $\dot{\varepsilon}_{0}^{V E}=0.01 s^{-1}, 0.02 s^{-1}, 0.03 s^{-1}, 0.04 s^{-1}$, local strain rates in the shoulders reach values of about $\dot{\varepsilon}=0.025 s^{-1}, 0.045 s^{-1}, 0.061 s^{-1}, 0.067 s^{-1}$ respectively. The results are reported in the same Fig.11 with curves F (filled squares) and F' (dashed line = Linear model). The linear regression of the data $\left(\mathrm{F}^{\prime}\right)$ clearly gives a line with a slope that falls between those obtained for $\varepsilon=1.2$ and $\varepsilon=1.4$. Additionally, the two points corresponding to the highest $\dot{\varepsilon}$ values still fall in the interval defined by the two linear models obtained for $\varepsilon=1.2$ and 
$\varepsilon=1.4$. This tends to validate the extrapolation of the linear behavior. Additionally, we checked that a quadratic dependency (another possible physical behavior) failed to encompass a global trend of these data (see Fig. C in Supplementary material).

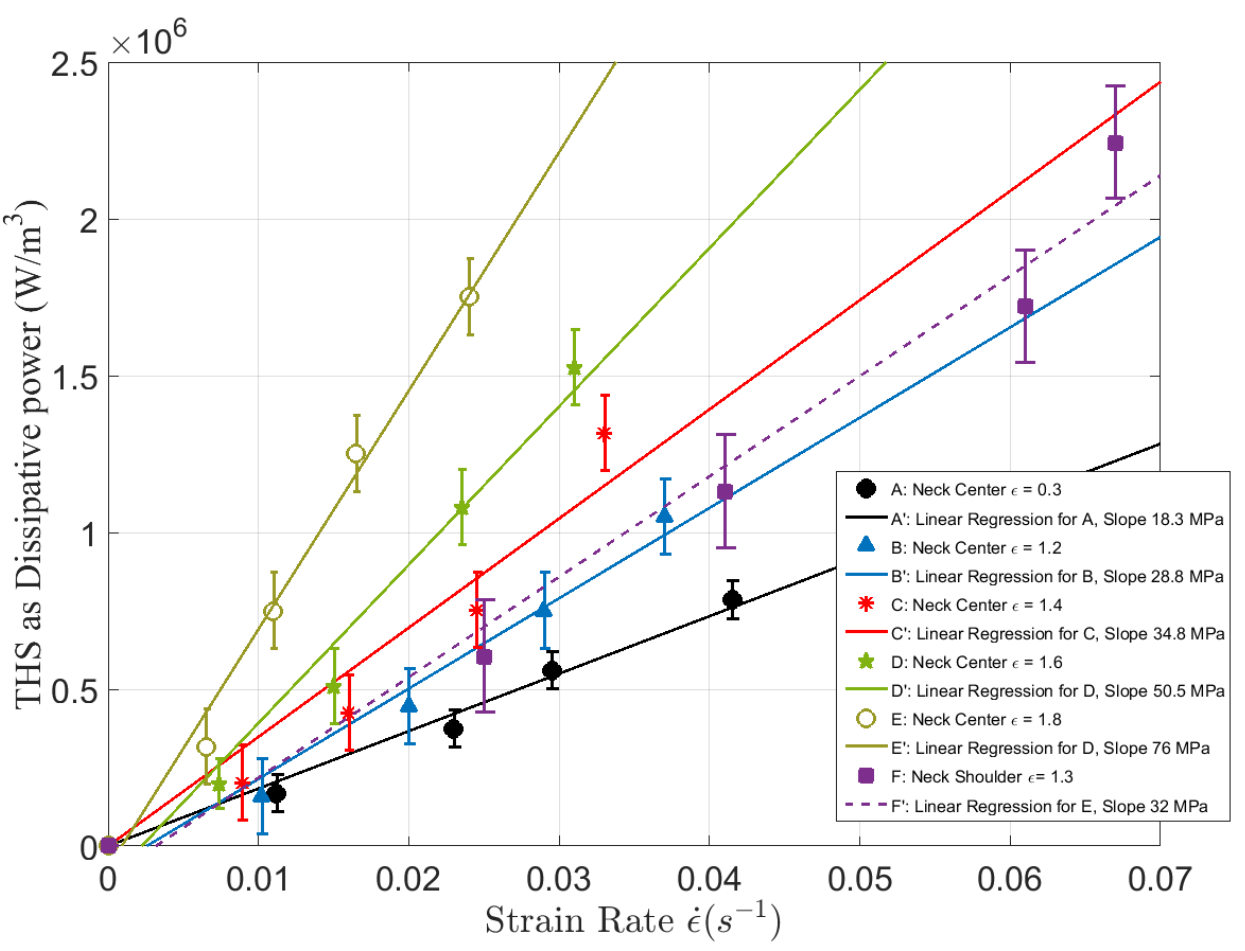

Fig. 11 THS versus strain rate as measured locally for 6 different strain states.

Rheological implications: THS measurements as a direct measurement of the flow stress All these data correspond to strains and strain rates associated with the necking development in a steady-state regime, beyond the onset of plastic instability but in the plastic regime of deformation, either in the primary stage ( single peak regime) or in the secondary stage (double-peak regime) when the plastic instability propagates in the specimen shoulders.

The linear dependency of $q_{T H S}^{\text {dissipative }}$ as function of the strain rate leads us to write $q_{\text {THS }}^{\text {dissipative }}=\sigma_{\varepsilon}^{\text {flow,THS }} \cdot \dot{\varepsilon}$ with the slopes (in $J / m^{3}$ or $P a$ ) associated to a "local" flow stress 
$\sigma_{\varepsilon}^{\text {flow,THS }}$ that depends on the local strain state: values given in the legend of Fig.11 and reported in Table 1 (line 2).

For a given microstructural state, we have then access to the local instantaneous stress producing the plastic deformation. This stress can in no way be assimilated to the yield stress as it is much less sensitive to strain rates in that range and almost equal to $\sigma^{\text {yield }} \simeq 33 \mathrm{MPa}$ obtained for example for the tensile test at $\dot{\varepsilon}_{0}^{V E}=0.01 s^{-1}$, see Fig.8. This value is very commonly obtained on HDPE (Ahmad, 1992). This thermomechanical approach made it clear that the macroscopic mechanical yield stress should not be mixed up with the thermomechanical flow stress. Where the yield stress corresponds to the critical stress necessary for the necking development of such polymers, the flow stress is directly connected to the heat dissipation produced locally by flow.

\begin{tabular}{|c|c|c|c|c|c|c|}
\hline Strain $\varepsilon$ & 0.3 & 1.2 & 1.3 & 1.4 & 1.6 & 1.8 \\
\hline $\begin{array}{c}\sigma_{\varepsilon}^{\text {flow,THS }}(M P a) \\
\text { (from fig.11) }\end{array}$ & 18.3 & 26.5 & 29.7 & 34.8 & 50.5 & 76 \\
\hline $\begin{array}{l}\sigma_{\varepsilon}^{\text {flow,TR }}(\mathrm{MPa}) \\
\text { (From Fig.12 and Model identification) }\end{array}$ & 17.5 & 28.1 & 30.3 & 40 & 54.2 & 78 \\
\hline
\end{tabular}

Table 1 Experimental values of $\sigma_{\varepsilon}^{\text {flow }}(M P a)$ obtained from THS measurements and form Tensile-Relaxation tests for different strain levels $\varepsilon .(=$ value obtained by linear interpolation between strains 1.2 and 1.4 )

We now show that the flow stress values obtained through THS measurements can be absolutely related to the stress relaxation decrease when drawing is stopped and a constant strain dwell is imposed. This way of measuring the flow stress is the natural consequence of the physical meaning of flow stress: the stress necessary to sustain further plastic deformation at a particular strain. For this, we refer to data from true stress-true strain curves obtained in a 
previous study (Blaise et al., 2012) for a Tensile-Relaxation test performed at a strain rate of $\dot{\varepsilon}_{0}^{V E}=0.005 s^{-1}$ with some values also being given for a strain rate of $\dot{\varepsilon}_{0}^{V E}=0.02 s^{-1}$. The tensile stage is stopped at various levels of an imposed strain $\varepsilon$ (Video-extensometry measurements at neck center). From this set of curves (Fig. 12), we measured the flow stress $\sigma_{\varepsilon}^{\text {flow } T R}$ to obtain the difference between the stress reached when the tensile stage was stopped at a given value of $\varepsilon$, and the asymptotic stress $\sigma^{r, \infty}$ reached for indefinite relaxation, also known as the relaxed stress. Both were obtained by an identification process performed on the whole curve due to the model detailed in Blaise et al., (2016) (See Fig. D in Supplementary material). The confidence bounds on $\sigma_{\varepsilon}^{\text {flow,TR }}$ were estimated classically, by computation of the sensitivity coefficients and variance-covariance matrix. They range from $\approx \pm 0.25 M P a$ for $\varepsilon=0.03$ to $\approx \pm 2 M P a$ for $\varepsilon=1.8$. In Table 1 (line 3 ) some specific values of $\sigma_{\varepsilon}^{\text {flow,TR }}$ to be compared to $\sigma_{\varepsilon}^{\text {flow,THS }}$ are reported. 


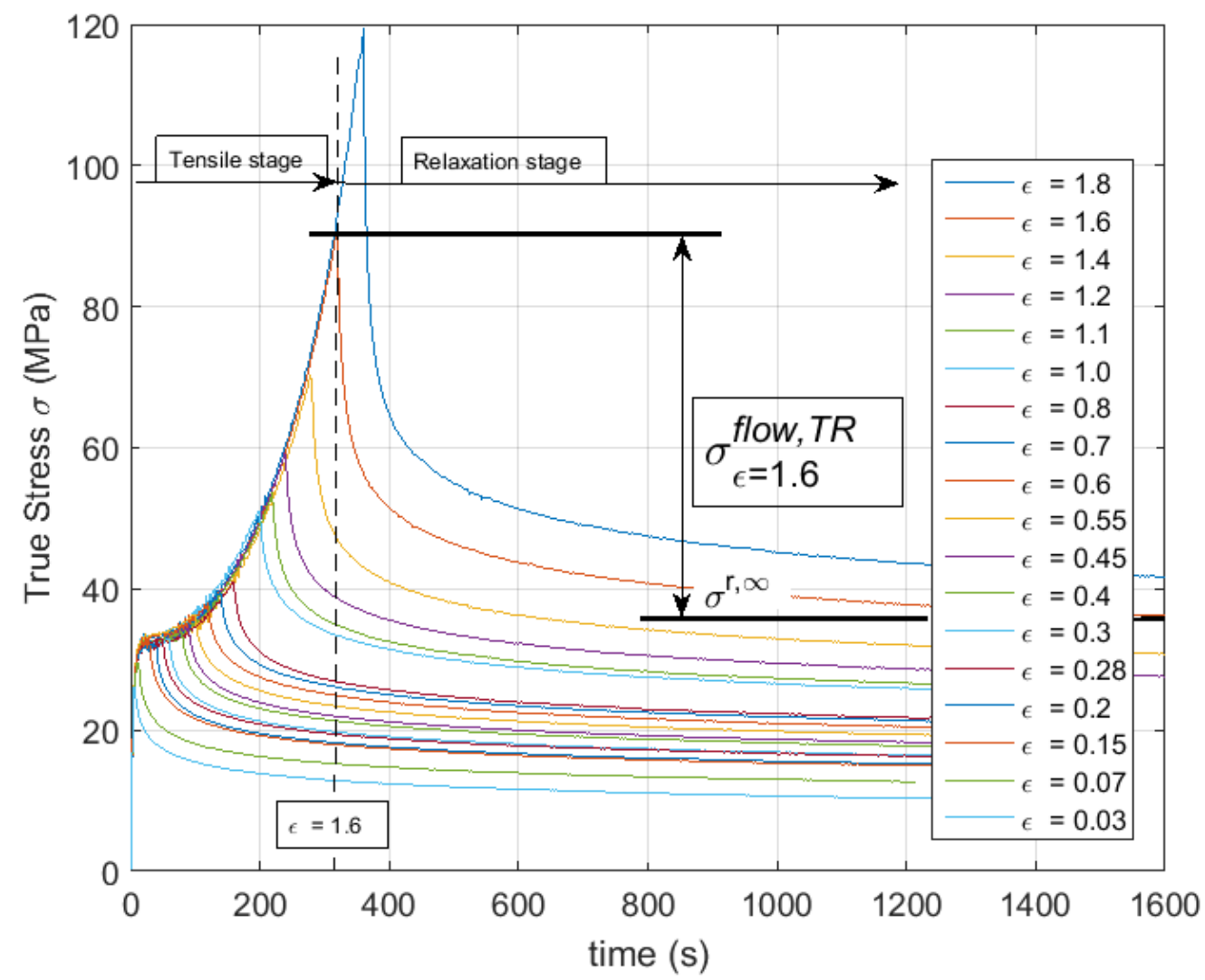

Fig. 12 Tensile tests followed by relaxations initiated at different $\varepsilon$ strains. 
Fig. 13 plots all available points (star dots) obtained in this manner. A fairly good correspondence between both flow stress estimations can then be observed. This original result makes it clear that the flow stress needed to make the material flow in a plastic regime (assimilated to neck propagation) is responsible for the internal heat dissipation. It would be interesting now to observe this rheological evidence on other systems (a purely amorphous material for example).

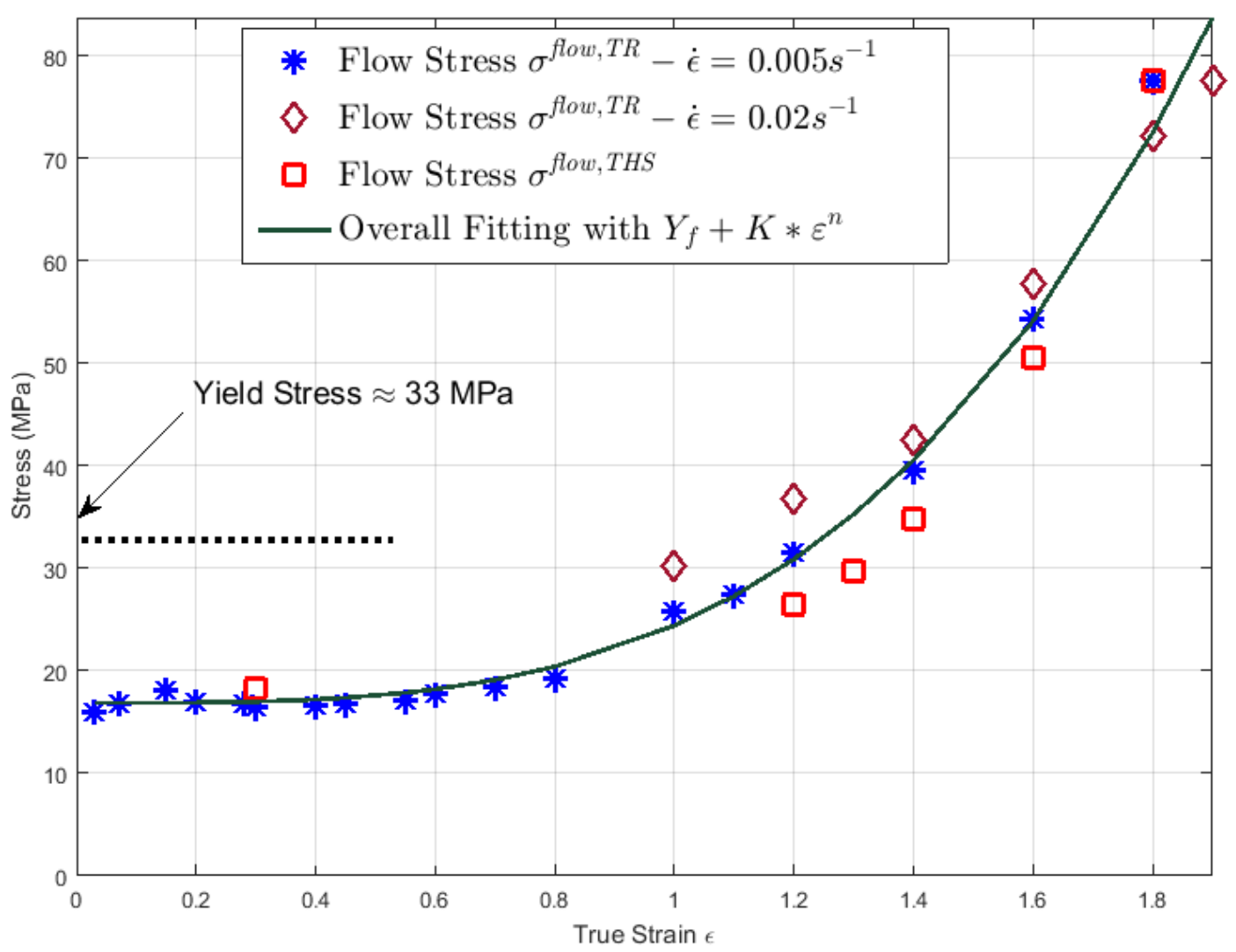

Fig. 13 Experimental values of $\sigma_{\varepsilon}^{\text {flow }}(\mathrm{MPa})$ obtained from THS measurements and from Tensile-Relaxation tests as a function of the strain, $\varepsilon$. Solid curve $=$ fitting all data with eq. $Y_{f}+K \varepsilon^{n}\left(Y_{f}=16.85 M P a, K=7.5 M P a, n=3.4\right)$. 


\section{Conclusion}

The experimental study reported here concerned the calorimetric effects occurring under the mechanical drawing of semi-crystalline polymers. This was shown to be a lengthy and tedious process. To obtain THS measurements from mechanical tests is tedious. It involves a good metrology, associated with complicated mathematical treatments such as inversion, and lastly a consistent and efficient thermodynamic model to account for couplings between rheological, thermal and microstructural behavior. Additionally, even for polymers in the glass transition regime, for which irreversible processes are in evidence, the strong thermomechanical couplings like those studied here, where the thermal effects are produced by the material itself, are very limited in magnitude. Strain rates above $0.01 s^{-1}$ were necessary in this study to induce measurable quantities, but were soon limited in their upper bound by the machine capacities and interest of using a non-adiabatic model for inversion. Future efforts in the direction of measuring calorimetric effects in specimens undergoing mechanical solicitation should therefore be considerably rethought.

Despite that point, THS measurements have been proved to be fully in phase with pure kinematical effects; namely THS behaves exactly like strain-rates. From that consideration, and trying to go further into the rheological consequences of that observation, we have shown that THS versus strain rate dependency leads to measurement of the flow stress associated with neck propagation, at least when THS can be considered as representative of the dissipated power i.e. internal entropy generation. The good comparison obtained with flow stress measured alternatively using simple tensile-relaxation tests, validates the rheological findings we made. It suggests that additional efforts from the community could reach calorimetric measurements of such a type in a more precise and direct way. 


\section{References}

Adams GW, Farris RJ (1988) Latent energy of deformation of bisphenol A Polycarbonate. J Polym Sci B Polym Phys 26:433-445.

Ahmad SH (1992) Flow stress of high density polyethylene and nylon 66 at high rates of strain, Polym Int 28(4): 291-294.

André S, Meshaka Y, Cunat C, (2003) Rheological constitutive equation of solids: a link between models based on irreversible thermodynamics and on fractional order derivative equations. Rheol Acta 42:500-515.

André S, Baravian C, Renault N, Cunat C, (2007) In situ mechanical characterization of polymers with the association of three optical techniques. Appl Phys Lett, 91(7):071919.

André S, Renault N, Meshaka Y, Cunat C (2012) From the thermodynamics of constitutive laws to thermomechanical experimental characterization of materials: An assessment based on inversion of thermal images. Continuum Mech Therm 24(1): 1-20.

Auffray N, Bonnet M, Pagano S, (2013) Identification of transient heat sources using the reciprocity gap. Inverse Probl Sci Eng 21(4): 721-738.

Bhalla KS, Zehnder AT, Han X, (2003), Thermomechanics of slow stable crack growth: closing the loop between experiments and computational modeling. Eng Fract Mech 70:2439-2458.

Blaise A, Andre S, Delobelle P, Meshaka Y, Cunat C, (2016), Advantages of a 3-parameter Reduced Constitutive Model for the Measurement of Polymers Elastic Modulus using Tensile Tests., Mech Time-Depend Mater 20:553-577.

Blaise A, Baravian C, Dillet J, Michot LJ, André S, (2011) Characterization of the mesostructure of HDPE under "in-situ" uniaxial tensile test by incoherent polarized steady-light transport, J Polym Sci B Polym Phys 50(5):328-337.

Chrysochoos A, Louche H, (2000) An infrared image processing to analyze the calorific effects accompanying strain localization. Int J Eng Sci 38:1759-1788.

Callen HB, (1985) Thermodynamics and an Introduction to Thermostatics, John Wiley \& Sons, New York.

Coussot P, (2018) Slow flows of yield stress fluids: yielding liquids or flowing solids?, Rheol Acta 57:1-14.

Cunat C, (2001) The DNLR approach and relaxation phenomena. Part I : Historical account and DNLR formalism. Mech Time-Depend Mater 5:39-65.

Delpueyo D, Balandraud X, Grédiac M, (2013) Heat source reconstruction from noisy temperature fields using an optimised derivative Gaussian filter. Infrared Phys Technol, 60:312-322.

De Sousa DM, Roberty NC, (2012) An inverse source problem for the stationary diffusion-advection-decay equation, Inverse Probl Sci Eng 20(7):891-915.

Doudard C, Calloch C, Hild F, Roux S, (2010) Identification of heat source from infrared thermography: Determination of 'self-heating' in a dual-phase steel by using a dog bone sample. Mech. Mater. 42:55-62. 
Farge L, Boisse J, Bihannic I, Diaz A, André S (2017), Anisotropy development during necking of HDPE studied at the microscale with in situ continuous 1D SAXS scans, J Polym Sci B Polym Phys 56(2): 170-181.

Kuiken GDC, (1994) Thermodynamics of Irreversible Processes: Applications to Diffusion and Rheology, Wiley Ch7, pp.239-299.

Maalej T, Maillet D, Fontaine J-R, (2012) Estimation of position and intensity of a pollutant source in channel flow using transmittance functions. Inverse Probl 28(5):055010.

Maj M, Oliferuk W, (2012) Analysis of plastic strain localization on the basis of strain and temperature fields. Arch. Metall. Mater. 57(4):1111-1116.

Meixner J, (1954) Thermodynamische theorie der elastichen relaxation. Z. Naturforschg 9a:654-663.

Münster A, (1966) Thermodynamique Des Processus Irréversibles. Institut National des Sciences et Techniques Nucléaires and Presses Universitaires de France, Paris-Saclay.

Nowick AS, Berry BS, (1972) Anelastic relaxation in crystalline solids, Academic Press, New York and London, pp 115-129.

Prigogine I, Defay R (1944-1946), Thermodynamique chimique conformément aux méthodes de Gibbs et De Donder, Tomes I-II. Gauthier-Villars.

Rap A, Elliott L, Ingham DB, Lesnic D, Wen X, (2007) The inverse source problem for the variable coefficients convection-diffusion equation, Inverse Problems in Science and Engineering 15(5):413-440. DOI: $10.1080 / 17415970600731274$

Rosenberg E, Brusselle-Dupend N, Epsztein T (2011) A mesoscale quantification method of cavitation in semicrystalline polymers using X-ray microtomography. Materials Science and Engineering A 528:6535-6544.

Salamatina O, Höhne G, Rudnev S, Oleinik E (1994) Work, heat and stored energy in compressive plastic deformation of glassy polymers. Thermochim. Acta 247:1-18.

Tauchert T, Afzal S, (1967) Heat generated during torsional oscillations of polymethylmethacrylate tubes. J Appl. Phys. 38(2):4568-4572.

Tisza L (1966) Generalized Thermodynamics. M.I.T. Press, Cambridge.

Toussaint E, Balandraud X, Le Cam JB, Grédiac M, (2012) Combining displacement, strain, temperature and heat source fields to investigate the thermomechanical response of an elastomeric specimen subjected to large deformations. Polym Test 31:916-925.

Videcoq E, Quemener O, Lazard M, Neveu A (2008), Heat source identification and on-line temperature control by a Branch Eigenmodes Reduced Model. Int. J. of Heat and Mass Transfer, 51, 4743-4752.

Ye J, Andre S, Farge L, (2015) Kinematic Study of Necking in a SemiCrystalline Polymer through 3D Digital Image correlation, Int J Solids Struct 59:48-72.

Ye J, Farge L, André S, Neveu A, (2016) A numerical study of heat source reconstruction for the advection-diffusion operator: A conjugate gradient method stabilized with SVD. Int J Therm Sci, 104:68-85. 


\section{SUPPLEMENTARY INFORMATION A}

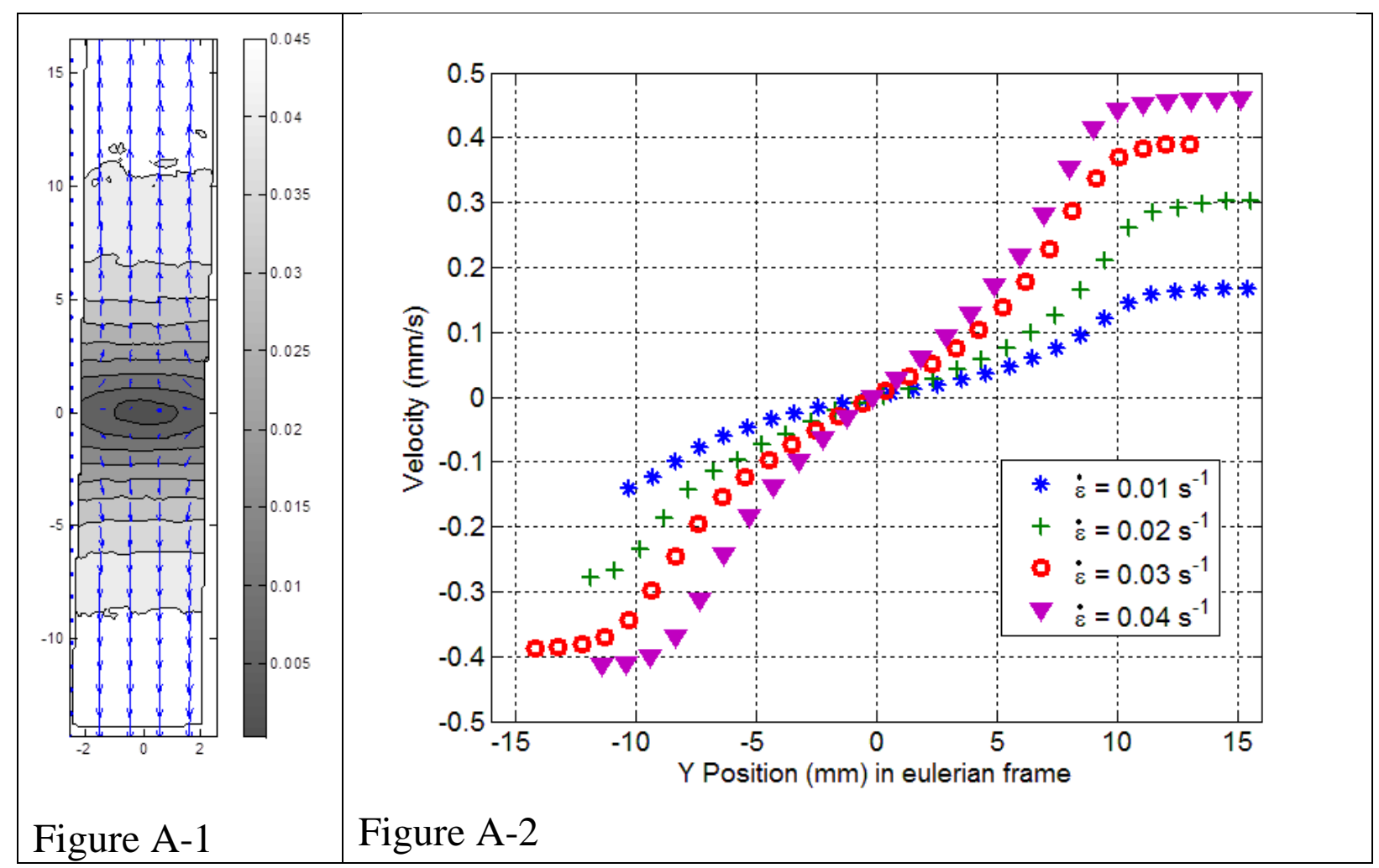

Figure A-1: Example of velocity field (vectors and magnitude contour plot in $\mathrm{mm} / \mathrm{s})$ for $\dot{\varepsilon}_{0}^{V E}=0.01 \mathrm{~s}^{-1}\left(\varepsilon_{0}^{V E}=0.12\right)$.

Figure A-2: Experimental velocity profiles measured with DIC for all strainrates and all strain states $\left(\varepsilon_{0}^{V E}=1.8\right)$

(from Ye et al., 2015) 


\section{SUPPLEMENTARY INFORMATION B}

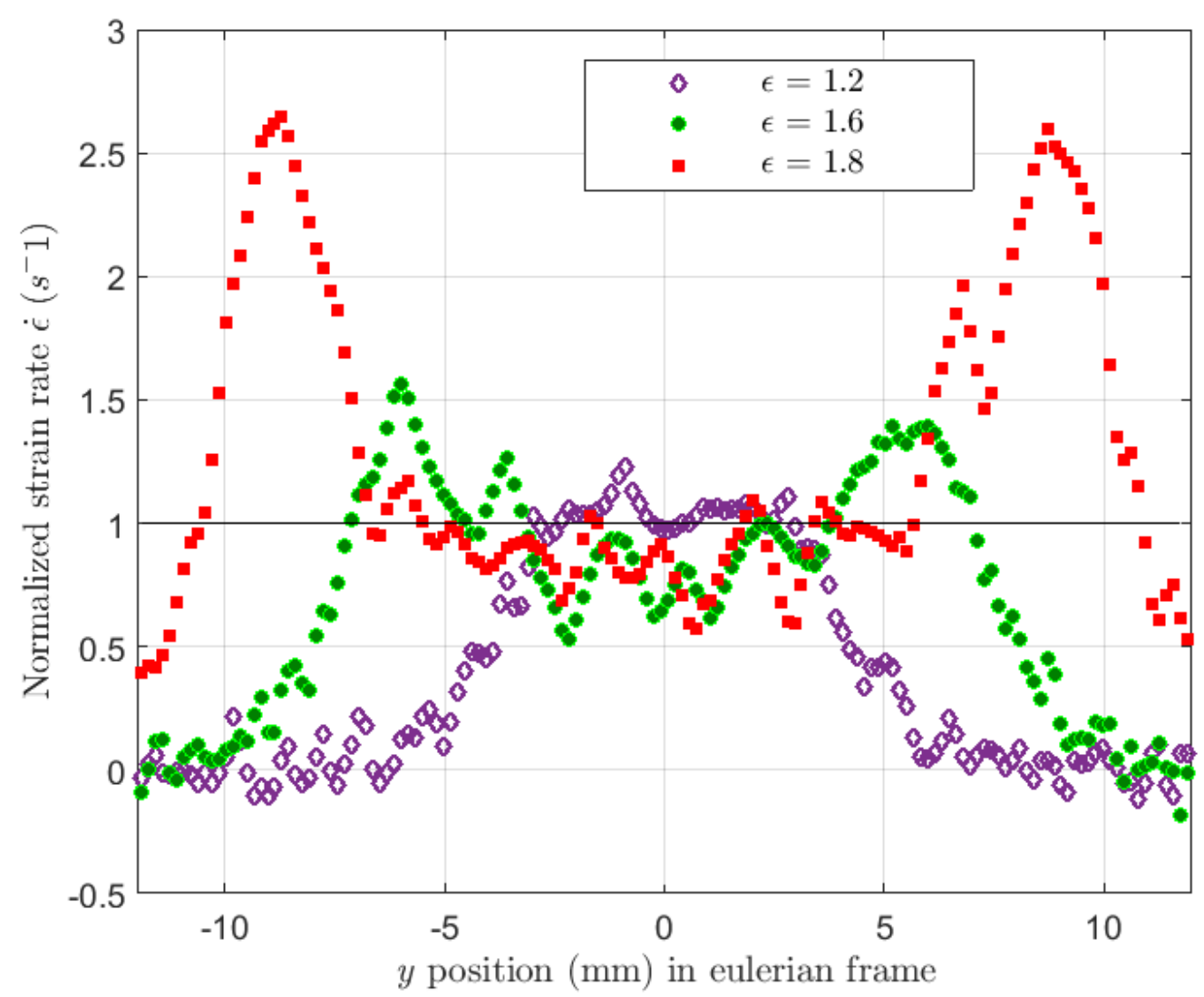

Figure B: Longitudinal profiles of the normalized strain rates $\dot{\varepsilon}$ for the 3 different strains $\varepsilon=1.2, \varepsilon=1.6, \varepsilon=1.8$ (Tensile test performed at $\dot{\varepsilon}=0.01 \mathrm{~s}^{-1}$ ) Field measurements performed by DIC.

The plateau present in the central part of the curves, of notable extent in eulerian coordinates, can clearly be seen, along with the symmetric double peaks at the striction's shoulder locations. The requested imposed strain rate in the neck center, ideally shown with a horizontal black line on the figure, is based on Video-extensometry. With this system, the gauge length is extended during the experiment, which produces at the end local strains below the targeted value. These have to be accounted for when considering local measurements in the specimen center, as is done for THS in Fig.11. For $\varepsilon=1.6$, or $\varepsilon=1.8$, the strain rate really imposed in the middle of the specimen is around $75 \%$ of the ideal target $\dot{\varepsilon}=0.01 \mathrm{~s}^{-1}$. Interpolation was performed to obtain precise values at $y=0$. 
SUPPLEMENTARY INFORMATION C

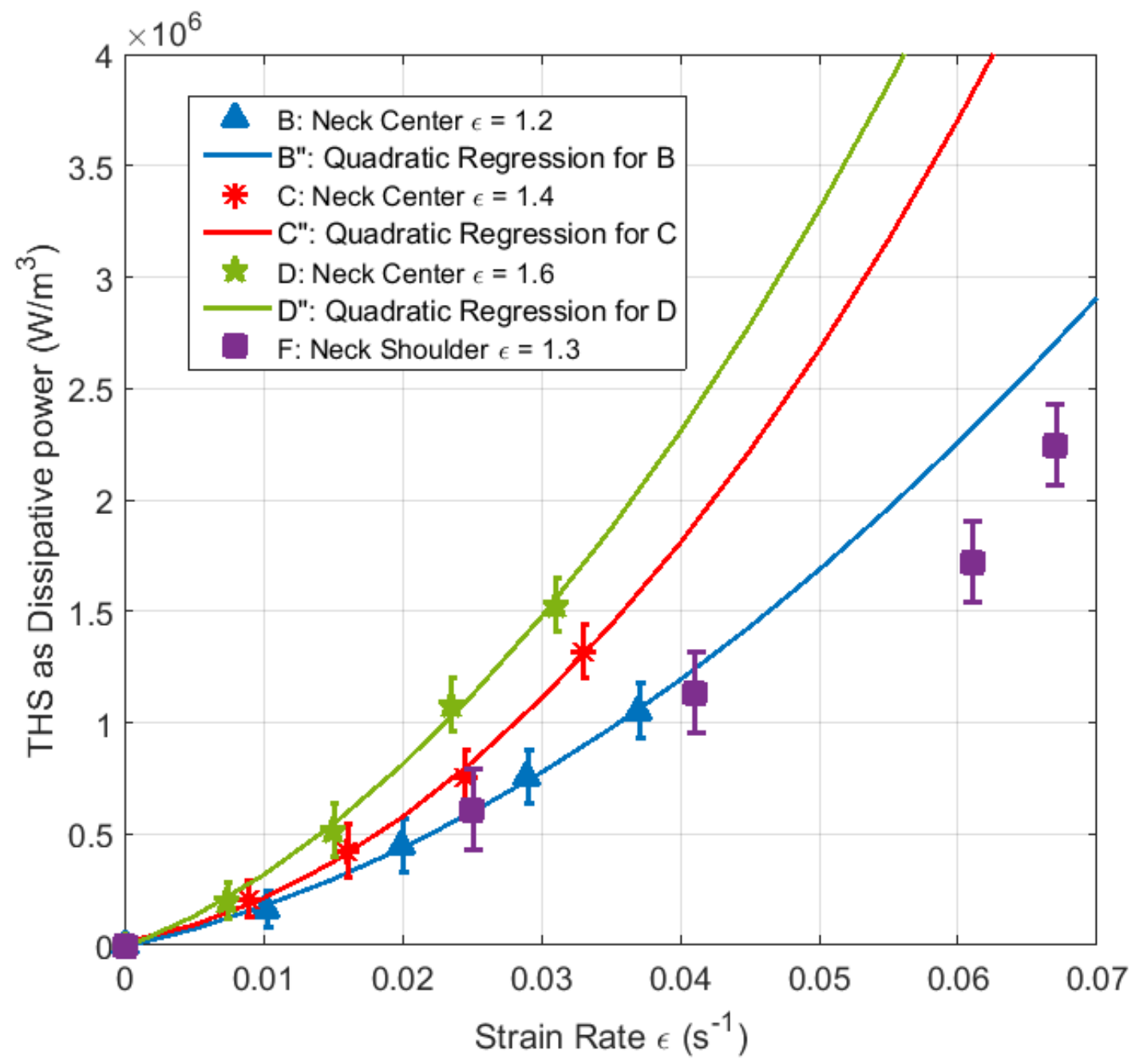

Figure C: THS versus strain rate with quadratic regression. For a local strain of 1.3 (square dots) obtained in the neck shoulders the quadratic model does not hold at high strain states. 


\section{SUPPLEMENTARY INFORMATION D}

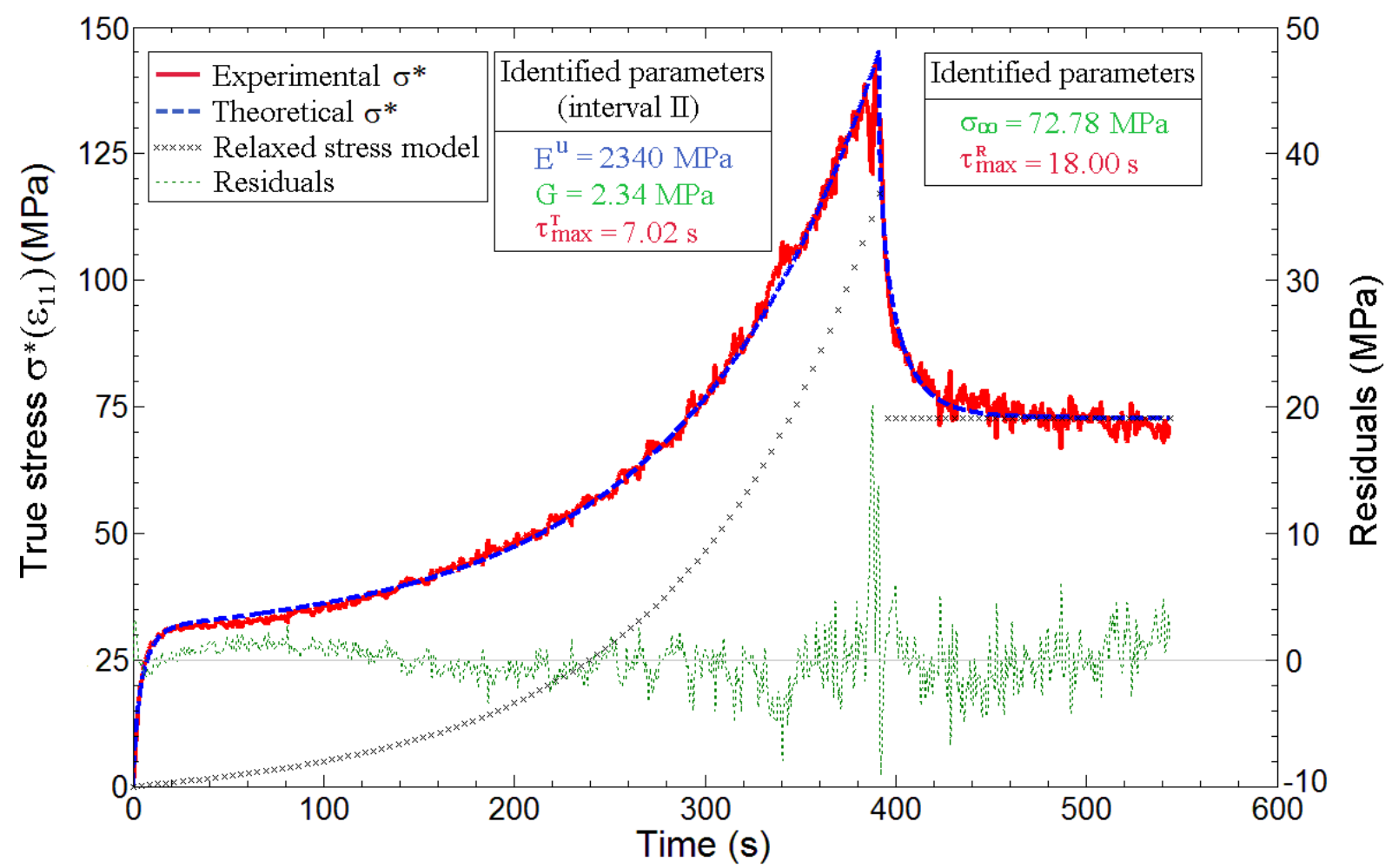

Figure D: Tensile test followed by a relaxation on HDPE specimen (Röchling Natural 500 Grade). Experimental data, fitted curve and residuals as a function of time - Strain rate of $5 \cdot 10^{-3} s^{-1}: 390 \mathrm{~s}$ corresponds to a true strain of 1.95 .

(Figure from Blaise et al., 2016)

The model described in Blaise et al., (2016) is used for the inverse determination of the model parameters limited to 5 :

- $\quad E^{u}$ instantaneous elastic modulus, a parameter identified over the whole tensile-relaxation time interval;

- $\quad G$ hardening modulus describing the relaxed state, identified on the tensile part of the curve;

- $\quad$ Maximum relaxation times $\tau_{\max }^{T}$ and $\tau_{\max }^{R}$ of a "universal" spectrum identified separately on the tensile and relaxation parts. When tension is stopped, the material is no longer the same and a new spectrum has to be considered for new viscoelastic behavior;

- $\quad \sigma_{\infty}$ : A new relaxed stress description (constant) for the relaxation stage.

The flow stress $\sigma_{\varepsilon}^{\text {flow,Relax }}$ is calculated in the article from the difference $\sigma_{\varepsilon}^{\text {flow, Relax }}=\sigma_{\varepsilon^{\text {elax }}}^{\text {max }}-\sigma_{\infty}$ both values being given as outputs of the parameter estimation process. In Fig. C, we have a flow stress $\sigma_{1.95}^{\text {flow, Relax }}=75.2 \mathrm{MPa}$ identified for $\varepsilon=1.95$. 